\title{
Learning by Investing, Embodiment, and Speed of Convergence
}

Groth, Christian; Wendner, Ronald

Publication date:

2011

Document version

Early version, also known as pre-print

Citation for published version (APA):

Groth, C., \& Wendner, R. (2011). Learning by Investing, Embodiment, and Speed of Convergence. Economic Policy Research Unit. Department of Economics, University of Copenhagen. 


\author{
Economic Policy Research Unit \\ Department of Economics \\ University of Copenhagen \\ $\emptyset$ ster Farimagsgade 5, Building 26 \\ DK-1353 Copenhagen K \\ DENMARK \\ Tel: (+45) 35324411 \\ Fax: (+45) 35324444 \\ Web: http://www.econ.ku.dk/epru/
}

\title{
Learning by Investing, Embodiment, and Speed of Convergence
}

Christian Groth, Ronald Wendner 


\title{
Learning by investing, embodiment, and speed of convergence
}

\author{
Christian Groth $^{a}$ and Ronald Wendner ${ }^{b}$ \\ ${ }^{a}$ Department of Economics and EPRU, University of Copenhagen, Denmark \\ ${ }^{b}$ Department of Economics, University of Graz, Austria
}

February 18, 2011

\begin{abstract}
This paper sets up a dynamic general equilibrium model to study how the composition of technical progress affects the asymptotic speed of convergence. The following questions are addressed: Will endogenizing a fraction of the productivity increases as coming from learning by investing help to generate a low asymptotic speed of convergence in accordance with the empirical evidence? Does it matter whether learning originates in gross or net investment? The answers to both questions turn out to be: yes, a lot. The third question addressed is: Does the speed of convergence significantly depend on the degree to which learning by investing takes the embodied form rather than the disembodied form? The answer turns out to be: no. These results point to a speed of convergence on the small side of $2 \%$ per year and possibly tending to a lower level in the future due to the rising importance of investment-specific learning in the wake of the computer revolution as the empirical evidence suggests.
\end{abstract}

Keywords and Phrases: Transitional dynamics, speed of convergence, learning by investing, embodied technological progress, decomposable dynamics.

JEL Classification Numbers: D91, E21, O41 


\section{Introduction}

The aim of this paper is to examine how the composition of technical progress affects the process of economic growth and in particular the asymptotic speed of convergence. We consider the composition of technical progress along three dimensions. The first relates to the source of technical change. We study the effect of the extent to which the source of technical change is learning from investment experience rather than exogenous. The second dimension relates to the basis of investment experience. Here we distinguish between the case where it is gross investment that adds to experience and the case where it is net investment. Although this distinction has not received much attention in the literature, it turns out to be very important. The third dimension relates to the degree to which technical change is embodied rather than disembodied. Following Solow (1960), technical change is said to be embodied if taking advantage of new technical knowledge requires construction of new investment goods. The newest technology is incorporated in the design of newly produced equipment; and this equipment will not participate in subsequent technical progress.

Two circumstances motivate our study of technical change along these dimensions. Available empirical research tends to come up with quite low estimates of the speed of convergence for industrialized economies. The influential inquiries by Barro and Sala-i-Martin (1992) and Mankiw et al. (1992) suggest annual rates of convergence of the order of 1.5 to 3 percent. The basic neoclassical growth model (the standard Ramsey model) when reasonably calibrated, however, yields rates of convergence of around 10 percent. This discrepancy fostered a theoretical literature (see below) showing how different extensions of the Ramsey model were capable of reducing the calibrated speed of convergence. The present paper adds to this literature by studying the questions: Will endogenizing productivity increases as coming primarily from learning by investing help to lower the model's implied speed of convergence? Does it matter whether learning originates in gross rather than net investment? The answers to both questions turn out to be affirmative. A quanti- 
tatively significant lowering of the asymptotic speed of convergence is involved.

The second circumstance that motivates our study is the seemingly increasing importance of embodiment of technical change in the wake of the computer revolution, as signified by a falling quality-adjusted relative price of capital equipment (Greenwood and Jovanovic 2001; Jovanovic and Rousseau, 2002; Hornstein et al., 2005). ${ }^{1}$ This gives rise to the question how a shift in the relative importance of disembodied and embodied technical progress is likely to affect the speed of convergence. Earlier theoretical literature (Phelps, 1962; Williams and Crouch, 1972) leads to the presumption that for a given aggregate rate of technical progress, a higher degree of embodiment results in faster convergence. Our study confirms this for the case where productivity growth stems from exogenous forces, but not for the case of growth driven by endogenous learning. Thus, we conclude that to the extent that embodied learning from gross investment explains a substantial part of a given per capita growth of $2 \%$ per year, the associated rate of convergence tends to be on the small side of $2 \%$ per year.

One of the first econometric studies of "conditional convergence" was accomplished by Barro and Sala-i-Martin (1992). To reconcile their finding of a speed of convergence of around $2 \%$ a year with the standard neoclassical growth model, an output elasticity with respect to capital as high as $0.75-0.8$ is needed. The authors suggest that such a high elasticity may be attained for "broad capital", based on adding physical and human capital. Ortigueira and Santos (1997) show that strictly convex capital installation costs tend to reduce the speed of convergence. Eicher and Turnovsky (1999) demonstrate that the speed of convergence is substantially reduced by adding an R\&D sector to the model. Turnovsky (2002) finds that the elasticity of substitution in production between capital and labor significantly affects the speed of convergence in the Ramsey model. A reduction in the elasticity of factor substitution from the benchmark level of one to a lower (empirically realistic) level, however, increases the model's implied speed of convergence and, thereby,

\footnotetext{
${ }^{1}$ An alternative, popular name for embodied technical change is investment-specific technical change.
} 
adds to the "convergence puzzle." Chatterjee (2005) argues that the convergence speed critically depends on capital utilization rates and that models with full capital utilization may overstate the speed of convergence.

To the list of theoretical factors reducing the speed of convergence, our paper adds an additional factor which seems both plausible and significant: embodied learning by investing.

After the influential econometric contributions by Barro and Sala-i-Martin (1992) and Mankiw et al. (1992), other studies have questioned their low estimates of the convergence speed, arguing that a number of econometric issues, like endogeneity of explanatory variables and country-specific fixed effects, have been ignored. Evidence has been put forward that the speed of convergence significantly varies across periods and groups of countries. Some studies provide estimates for a convergence speed of approximately $6 \%$ (Evans, 1997) and of $4.7 \%$ for a sample of 75 countries and $9.3 \%$ for OECD countries (Islam, 1995). Recently, the cross-country study by McQuinn and Whelan (2007), based on data for changes in the capital-output ratio, suggests convergence speeds of about $7 \%$ per year. On the other hand, based on calibration and an industry-related approach, Jovanovic and Rousseau (2002) find signs that the expansion of the applications of computers (the general-purpose technology of modern times) is likely to result in lower aggregate convergence speed and faster aggregate productivity growth than earlier general-purpose technologies like electricity and internal combustion.

Irrespective of the disagreement about the correct estimate of the convergence speed and whether there is a puzzle or not, the question how different factors affect the convergence speed is of interest. The speed of convergence is an indicator for the emphasis that should be placed on transitional dynamics of a growth model relative to the steady-state behavior. If for instance the speed of convergence is likely to decline in the future, then the transitional dynamics become more important for evaluating the effects of growth-promoting policies.

To examine how the composition of technical progress affects the speed of con- 
vergence, we set up a dynamic general equilibrium model, in continuous time, of embodied technical change in two versions, depending on whether the source of learning is gross or net investment. The basic framework is in the tradition of the path-breaking paper by Greenwood et al. (1997) on investment-specific technical change. By introducing endogenous learning from investment, our model essentially follows one of the "future directions" suggested by these authors. We depart, however, by allowing learning to imply scale effects on productivity levels. Such effects seem plausible in view of spillovers and the non-rival character of knowledge. On the other hand, we simplify by ignoring structures. We focus on the robust case of semi-endogenous growth rather than the knife-edge case of fully endogenous growth.

This focus, together with our multi-facetted description of technical change, is also the main difference vis-a-vis one of the models, named "Solow (1960) meets Arrow (1962)", in Greenwood and Jovanovic (2001). In relation to the theoretical part of the above-mentioned paper by Jovanovic and Rousseau (2002), our model differs by allowing multi-facetted technical change with learning based on gross rather than net investment and by assuming strictly concave utility (so that the interest rate is not fixed). The overall difference from the above-mentioned investmentspecific technical change papers is our primary focus on transitional dynamics and in particular the asymptotic speed of convergence.

The rest of the paper is organized as follows. Section 2 develops the grossinvestment based version of the model, which we refer to as the "benchmark model". This version leads to a three-dimensional dynamic system the steady-state and stability properties of which are studied in the first part of Section 3. The second part of Section 3 shows the novel result, linked to the distinction between decomposable and indecomposable dynamics, that as soon as learning from gross investment becomes part of the growth engine, the asymptotic speed of convergence displays a discrete fall. Section 4 describes the case of learning based on net investment. This "alternative model" leads to two-dimensional dynamics and the appealing discon- 
tinuity disappears. By numerical simulations, Section 5 quantifies the mentioned discontinuity implied by the benchmark model. In addition, Section 5 explores the otherwise smooth dependency of the speed of convergence on the composition of technical change along the three dimensions described above. Finally, Section 6 concludes.

\section{A benchmark model}

\subsection{Disembodied and embodied learning by investing}

The learning-by-investing hypothesis is that variant of the learning-by-doing hypothesis that sees the source of learning as being primarily experience in the investment goods sector. This experience embraces know-how concerning how to produce the capital goods in a cost-efficient way and how to design them so that in combination with labor they are more productive in their applications. The simplest model exploring this hypothesis is in textbooks sometimes called the Arrow-Romer model and is a unified framework building on Arrow (1962) and Romer (1986). The key parameter is a learning parameter which in the "Arrow case" is less than one and in the "Romer case" equals one. ${ }^{2}$ Whatever the size of the learning parameter, the model assumes that learning generates non-appropriable new knowledge that via knowledge spillovers across firms provides an engine of productivity growth for the major sectors of the economy. Summaries of the empirical evidence for learning and spillovers is contained in Jovanovic (1997) and Greenwood and Jovanovic (2001).

In the Arrow-Romer model firms benefit from recent technical advances irrespective of whether their equipment is new or old. That is, technical change is assumed to be disembodied: new technical knowledge improves the combined productivity of capital and labor independently of whether the workers operate old or new machines. No new investment is needed to take advantage of the recent technological or organizational developments.

In contrast we say that technical change is embodied, if taking advantage of new

\footnotetext{
${ }^{2}$ See, e.g., Valdés (1999) and Barro and Sala-i-Martin (2004).
} 
technical knowledge requires construction of new investment goods. The newest technology is incorporated in the design of newly produced equipment; and this equipment will not participate in subsequent technical progress. An example: only the most recent vintage of a computer series incorporates the most recent advance in information technology. In this way investment becomes an important bearer of the productivity increases which this new knowledge makes possible. This view is consistent with the finding in the cross-country study by Levine and Renelt (1992) that among over 50 different regressors, only the share of investment in GDP, other than initial income, is found to be strongly correlated with growth.

Let the aggregate production function be

$$
Y_{t}=K_{t}^{\alpha}\left(A_{t} L_{t}\right)^{1-\alpha}, \quad 0<\alpha<1
$$

where $Y_{t}$ is output, $L_{t}$ labor input, and $A_{t}$ labor-augmenting productivity originating in disembodied technical change, all at time $t$. Time is continuous. We consider two sources of growth in $A_{t}$, an endogenous source, investment experience, represented by the variable $J_{t}$, and an unspecified exogenous source, $e^{\gamma t}$ :

$$
A_{t}=J_{t}^{\beta} e^{\gamma t}, 0 \leq \beta<1, \gamma \geq 0
$$

The parameter $\beta$ indicates the elasticity of labor-augmenting productivity w.r.t. investment experience and is thus a measure of the strength of disembodied learning. For short we name $\beta$ the disembodied learning parameter. The upper bound on $\beta$ is brought in to avoid explosive growth. In our benchmark model we assume that investment experience, $J_{t}$, is proportional to cumulative aggregate gross investment,

$$
J_{t}=\int_{-\infty}^{t} I_{\tau} d \tau,
$$

where $I_{\tau}$ is aggregate gross investment at time $\tau$ and we have normalized the factor of proportionality to one. The parameter $\gamma$ in (2) is the rate of exogenous disembodied technical progress.

We consider a closed economy so that national income accounting implies

$$
Y_{t}=I_{t}+C_{t}
$$


where $C_{t}$ is aggregate consumption. We shall assume that, once produced, capital goods can never be used for consumption. So gross investment, $I_{t}$, is always nonnegative.

Based on data for the U.S. 1950-1990, Greenwood et al. (1997) estimate that embodied technical progress explains about $60 \%$ of the growth in output per man hour, the remaining $40 \%$ being accounted for by disembodied technical progress. So, empirically, embodied technical progress seems to play the dominant role. ${ }^{3}$ In line with Greenwood et al. (1997) we model embodied technical change in the following way:

$$
\dot{K}_{t}=Q_{t} I_{t}-\delta K_{t}, \quad \delta>0
$$

where a dot over a variable indicates the time derivative, and $Q_{t}$ measures investmentaugmenting productivity, for short just the "quality", of newly produced investment goods. The growing level of technology implies rising $Q_{t}$. A given level of investment thus gives rise to a greater and greater addition to the capital stock, $K_{t}$, measured in constant efficiency units. For realism and to allow a difference between gross and net investment we have the rate, $\delta$, of physical capital depreciation strictly positive.

As for growth in $A_{t}$, there are also two potential sources of growth in $Q_{t}$. One is an endogenous source in the form of investment experience, $J_{t}$. The other is an exogenous source, here represented by the factor $e^{\psi t}$. Specifically, we assume that

$$
Q_{t}=J_{t}^{\lambda} e^{\psi t}, \quad 0 \leq \lambda<\frac{1-\alpha}{\alpha}(1-\beta), \psi \geq 0 .
$$

That is, the quality $Q_{t}$ of investment goods of the current vintage is determined by cumulative experience which in turn reflects cumulative aggregate gross investment. The parameter $\lambda$ indicates the elasticity of the quality of newly produced investment goods w.r.t. investment experience and is thus a measure of the strength of embodied learning. For short we name $\lambda$ the embodied learning parameter. The upper bound on $\lambda$ is brought in to avoid explosive growth.

\footnotetext{
${ }^{3}$ Based on more recent data and partly different measurement methods Jovanovic and Rousseau (2002) and Sakellaris and Wilson (2004) reach even higher estimates of the importance of embodied technical change. For a survey, see Hornstein et al. (2005).
} 
TABLE 1

PARAMETERS CHARACTERIZING TECHNICAL CHANGE

\begin{tabular}{l|cc}
\hline \hline & \multicolumn{2}{|c}{ Source of technical change } \\
Form of technical change & Exogenous & Learning \\
\hline Disembodied & $\gamma$ & $\beta$ \\
Embodied & $\psi$ & $\lambda$ \\
\hline
\end{tabular}

Table 1 summarizes how the elasticity parameters relate to the source and the form, respectively, of technical progress. The third dimension of technical change that is in focus in this paper relates to whether the basis of investment experience is cumulative gross investment or net investment. This distinction can also be represented as a variation in the value of a parameter. We may introduce a rate of "experience depreciation", $\delta_{E}$, in (3) by replacing $I_{\tau}$ with $I_{\tau}-\delta_{E} J_{\tau}$. For simplicity this paper concentrates on two particular cases: $\delta_{E}=0$ and $\delta_{E}=\delta$. As the model structure is rather different in these two cases, we treat them separately, namely as the present "benchmark model" and the "alternative model" of Section 4 , respectively.

We now embed the described technology in a market economy with perfect competition where learning effects appear as externalities. That is, each firm is too small to have any recognizable effect on $A_{t}$ and $Q_{t}{ }^{4}$

Let the output good be the numeraire. The representative firm chooses inputs so as to maximize the profit $\Pi_{t}=K_{t}^{\alpha}\left(A_{t} L_{t}\right)^{1-\alpha}-R_{t} K_{t}-w_{t} L_{t}$, where $R_{t}$ is real cost per unit of capital services (the rental rate) and $w_{t}$ is the real wage. Given equilibrium in the factor markets, the rental rate must satisfy

$$
R_{t}=\alpha \tilde{k}_{t}^{\alpha-1}=\alpha \frac{Y_{t}}{K_{t}}
$$

where $\tilde{k}_{t}$ is the effective capital-labor ratio, $K_{t} /\left(A_{t} L_{t}\right)$, as given from the supply side. We assume labor supply is inelastic and grows at the constant rate $n \geq 0$.

\footnotetext{
${ }^{4}$ This view of learning as a pure externality is of course a simplification. In practice firms' investment decisions bear in mind that adoption of new technology takes time and requires learning. The productivity slowdown in the 1970s has by some been seen as reflecting not a slowdown in the pace of technical progress but rather a speed-up in embodied technical change resulting in a temporary productivity delay (see, e.g., Hornstein and Krusell, 1996).
} 
Since $Q_{t}$ units of the capital good can be produced at the same minimum cost as one unit of the consumption good, the equilibrium price of the capital good in terms of the consumption good is

$$
p_{t}=\frac{1}{Q_{t}}
$$

Denoting the real interest rate in the market for loans, $r_{t}$, we have the no-arbitrage condition

$$
\frac{R_{t}-\left(\delta p_{t}-\dot{p}_{t}\right)}{p_{t}}=r_{t}
$$

where $\delta p_{t}-\dot{p}_{t}$ is the true economic depreciation of the capital good per time unit. So, given the interest cost, $p_{t} r_{t}$, the rental rate (or user cost) of capital is higher, the faster $p_{t}$ falls, that is, the faster the quality of investment goods rises.

\subsection{Dynamics of the production sector}

From now the dating of the variables is suppressed when not needed for clarity. Let the growth rate of an arbitrary variable $x>0$ be denoted $g_{x} \equiv \dot{x} / x$. Let $z$ and $x$ denote the output-capital ratio and the consumption-capital ratio, respectively, both in value terms, that is, $z \equiv Y /(p K)$ and $x \equiv C /(p K)$. Then, substituting (4) into (5), the growth rate of capital can be written

$$
g_{K}=z-x-\delta
$$

In view of (8), $g_{p}=-g_{Q}$, and so, using (1), the growth rate of the output-capital ratio in value terms can be written

$$
g_{z}=g_{Y}-g_{p}-g_{K}=(\alpha-1) g_{K}+(1-\alpha)\left(g_{A}+n\right)+g_{Q},
$$

where

$$
\begin{aligned}
& g_{A}=\beta g_{J}+\gamma, \\
& g_{Q}=\lambda g_{J}+\psi,
\end{aligned}
$$


and $n \geq 0$ is the constant growth rate of the labor force (full employment is assumed). By taking the time derivative on both sides of (3) we get $\dot{J}=I$ so that

$$
g_{J}=\frac{I}{J} \equiv s u
$$

where $s$ is the saving-output ratio, i.e., $s \equiv I / Y \in[0,1]$, and $u$ is the outputexperience ratio, i.e., $u \equiv Y / J$.

It follows that

$$
g_{z}=(\alpha-1)(z-x-\delta)+[(1-\alpha) \beta+\lambda] s u+(1-\alpha)(\gamma+n)+\psi,
$$

and

$$
g_{u}=g_{Y}-g_{J}=\alpha(z-x-\delta)+[(1-\alpha) \beta-1] s u+(1-\alpha)(\gamma+n),
$$

where we have applied (1), (10), (11), (12), and (13). In these two equations we can substitute $s \equiv I / Y=1-x / z$, by (4) and the definitions of $x$ and $z$. As a result the dynamics of the production sector is described in terms of the three endogenous variables $z, x$, and $u$. The role of the household sector is represented by $x$, which depends on households' consumption.

\section{$2.3 \quad$ A representative household}

The representative household has $L_{t}$ members, each supplying one unit of labor inelastically per time unit. As indicated above, the growth rate of $L_{t}$ is $n$. The household has a constant rate of time preference $\rho>0$ and an instantaneous CRRA utility function with absolute elasticity of marginal utility of consumption equal to $\theta>0$. Facing given market prices and equipped with perfect foresight the household chooses a plan $\left(c_{t}\right)_{t=0}^{\infty}$ so as to

$$
\begin{array}{rlr}
\max U_{0} & =\int_{0}^{\infty} \frac{c_{t}^{1-\theta}}{1-\theta} L_{t} e^{-\rho t} d t & \text { s.t. } \\
\dot{V}_{t} & =r_{t} V_{t}+w_{t} L_{t}-c_{t} L_{t}, \quad V_{0} \text { given, and } \\
\lim _{t \rightarrow \infty} V_{t} e^{-\int_{0}^{t} r_{s} d s} & \geq 0,
\end{array}
$$


where $c \equiv C / L$ is per capita consumption, $V=p K$ is financial wealth, and (18) is the No-Ponzi-Game condition. ${ }^{5}$ Again, letting the dating of the variables be implicit, an interior solution satisfies the Keynes-Ramsey rule,

$$
\frac{\dot{c}}{c}=\frac{1}{\theta}(r-\rho)=\frac{1}{\theta}\left(\alpha z-\delta-g_{Q}-\rho\right),
$$

and the transversality condition that the No-Ponzi-Game condition holds with strict equality:

$$
\lim _{t \rightarrow \infty} V_{t} e^{-\int_{0}^{t} r_{s} d s}=0 .
$$

The last equality in (19) follows from (9), (8), and (7).

Before proceeding, it is worth to briefly compare the present model with related models in the literature, not already mentioned in the introduction. Embodied learning from investment is also a growth-driving force in one of the models in Groth et al. (2010) and the model in Groth (2010). These papers, however, more or less leave out the interplay with additional growth-driving factors and the focus is on other aspects of growth than adjustment speed. The present model is also related to the investment-specific learning model by Boucekkine et al. (2003). Like the textbook Arrow-Romer model referred to above, however, Boucekkine et al. (2003) assume that learning derives from net investment. A precise comparison with their approach is therefore better handled in connection with the "alternative model" of Section 4 below.

\section{The implied dynamic system}

Log-differentiating the consumption-capital ratio $x=c L /(p K)$ w.r.t. $t$ and applying (19) and (8) gives

$$
\begin{aligned}
g_{x} & =\frac{1}{\theta}\left(\alpha z-\delta-g_{Q}-\rho\right)+n+g_{Q}-g_{K} \\
& =\frac{1}{\theta}(\alpha z-\delta-\rho)-(z-x-\delta)+n+\left(1-\frac{1}{\theta}\right)(\lambda s u+\psi),
\end{aligned}
$$

where $s \equiv 1-x / z$.

\footnotetext{
${ }^{5}$ In case $\theta=1$, the instantaneous utility function in (16) should be interpreted as $\ln c_{t}$.
} 
The dynamics of the economy are described by the three differential equations, (21), (14), and (15), in the endogenous variables, $x, z$, and $u$. There are two predetermined variables, $z$ and $u$, and one jump variable, $x$. A (non-trivial) steady state of the system is a point $\left(x^{*}, z^{*}, u^{*}\right)$, with all coordinates strictly positive, such that $(x, z, u)=\left(x^{*}, z^{*}, u^{*}\right)$ implies $\dot{x}=\dot{z}=\dot{u}=0 .{ }^{6}$ We now study existence and properties of such a steady state.

\subsection{Steady state}

The economy will in steady state follow a balanced growth path (BGP for short), defined as a path along which $K, Q, Y$, and $c$ grow at constant rates, not necessarily positive. To ensure positive growth we need the assumption

$$
\gamma+\psi+n>0
$$

This requires that at least one of these nonnegative exogenous parameters is strictly positive. Moreover, it turns out that this is needed to ensure that a viable economy (one with $Y>0$ ) can be situated in a steady state.

In steady state we have $g_{u}=0$. So by definition of $u$ we get $g_{Y}^{*}=g_{J}^{*}=s^{*} u^{*}$ from (13). By setting the right-hand sides of (14) and (15) equal to nil and solving for $g_{Y}^{*}\left(=s^{*} u^{*}\right)$ and $g_{K}^{*}\left(=z^{*}-x^{*}-\delta\right)$ we thus find

$$
g_{Y}^{*}=s^{*} u^{*}=\frac{\alpha \psi+(1-\alpha)(\gamma+n)}{(1-\alpha)(1-\beta)-\alpha \lambda}>0
$$

and

$$
g_{K}^{*}=\frac{[1-(1-\alpha) \beta] \psi+(1+\lambda)(1-\alpha)(\gamma+n)}{(1-\alpha)(1-\beta)-\alpha \lambda}>0 .
$$

That the two growth rates are strictly positive is due to (A1) combined with the restriction imposed in (6) on the embodied learning parameter $\lambda$. We see that $g_{K}^{*} \geq$ $g_{Y}^{*}$ always. Strict inequality holds if and only if $\psi$ (embodied exogenous technical change) or $\lambda$ (embodied learning) is positive. ${ }^{7}$ Thus, when technical progress has

\footnotetext{
${ }^{6}$ Generally, steady state values of variables will be marked by an asterisk.

${ }^{7}$ We have $1-(1-\alpha) \beta>\alpha$ in view of $\alpha, \beta \in(0,1)$.
} 
an embodied component, $K$ grows faster than $Y$. This outcome is in line with the empirical evidence presented in, e.g., Greenwood et al. (1997).

According to (12), (13), and (22),

$$
g_{Q}^{*}=\frac{(1-\alpha)[(1-\beta) \psi+\lambda(\gamma+n)]}{(1-\alpha)(1-\beta)-\alpha \lambda} .
$$

Given (A1), we have $g_{Q}^{*}>0$ if and only if $\psi$ (embodied exogenous technical change) or $\lambda$ (embodied learning) is positive. A mirror image of this is that the price $p$ $(\equiv 1 / Q)$ of the capital good in terms of the consumption good is falling whenever there is embodied technical progress. Indeed,

$$
g_{p}^{*}=-g_{Q}^{*}=-\frac{(1-\alpha)[(1-\beta) \psi+\lambda(\gamma+n)]}{(1-\alpha)(1-\beta)-\alpha \lambda} .
$$

Whether or not $Y / K$ is falling, the output-capital ratio in value terms, $Y /(p K)=z^{*}$, stays constant along a BGP.

By constancy of $x^{*} / z^{*}=(c L / Y)^{*}$ we conclude that $c L$ is proportionate to $Y$ in steady state. Hence $g_{c}^{*}=g_{Y}^{*}-n$ so that, combining (19) and (22), we find

$$
g_{c}^{*}=\frac{1}{\theta}\left(\alpha z^{*}-\delta-g_{Q}^{*}-\rho\right)=\frac{(1-\alpha) \gamma+\alpha \psi+[(1-\alpha) \beta+\alpha \lambda] n}{(1-\alpha)(1-\beta)-\alpha \lambda}>0,
$$

where the inequality is due to (A1). The learning processes, whether in disembodied or embodied form, represented by $\beta$ and $\lambda$, respectively, create and diffuse a nonrival good, technical knowledge. So learning by investing brings about a tendency to increasing returns to scale in the system. The way $n$ appears in (26) indicates that the positive effect of $\beta$ and $\lambda$ on the growth rate of per capita consumption gets a boost via interaction with an expanding labor force, which signifies a rising scale of the economy. ${ }^{8}$ In contrast, the disembodied and embodied exogenous sources of productivity growth, represented by $\gamma$ and $\psi$, respectively, affect per capita growth independently of growth in the labor force.

\footnotetext{
${ }^{8}$ In view of cross-border technology diffusion, the growth-enhancing role of labor force growth inherent in knowledge-based growth models should not be seen as a prediction about individual countries in an internationalized world, but rather as pertaining to larger regions, perhaps the world economy.
} 
To ensure boundedness of the discounted utility integral we shall throughout impose the parameter restriction

$$
\rho-n>(1-\theta) \frac{(1-\alpha) \gamma+\alpha \psi+[(1-\alpha) \beta+\alpha \lambda] n}{(1-\alpha)(1-\beta)-\alpha \lambda} .
$$

This condition is equivalent to $\rho-n>(1-\theta) g_{c}^{*}$.

From (26) and (24) we find

$$
\begin{aligned}
z^{*}= & \frac{[(1-\alpha) \gamma+\alpha \psi] \theta+(1-\alpha)[\lambda \gamma+(1-\beta) \psi]+\{[(1-\alpha) \beta+\alpha \lambda] \theta+(1-\alpha) \lambda\} n}{\alpha[(1-\alpha)(1-\beta)-\alpha \lambda]} \\
& +\frac{\rho+\delta}{\alpha}>0 .
\end{aligned}
$$

By (10), the steady state value of the consumption-capital ratio is $x^{*}=z^{*}-g_{K}^{*}-$ $\delta$; into this expression (27) and (23) can be substituted (the resulting formula is huge, cf. Appendix A). The saving rate in steady state is $s^{*}=1-x^{*} / z^{*}>0$ (see Proposition 1 below). By substituting this into (22) we get the output-experience ratio as $u^{*}=g_{Y}^{*} / s^{*}$.

Finally, by (19) the real interest rate in steady state is

$$
r^{*}=\alpha z^{*}-\delta-g_{Q}^{*}=\theta g_{c}^{*}+\rho=\theta \frac{(1-\alpha) \gamma+\alpha \psi+[(1-\alpha) \beta+\alpha \lambda] n}{(1-\alpha)(1-\beta)-\alpha \lambda}+\rho .
$$

The parameter restriction (A2) ensures that the transversality condition of the household is satisfied in the steady state. Indeed, from (A2) we have $r^{*}=\theta g_{c}^{*}+\rho$ $>g_{c}^{*}+n=g_{Y}^{*}=g_{p}^{*}+g_{K}^{*}=g_{V}^{*}$ since $z \equiv Y /(p K) \equiv Y / V=z^{*}$ in steady state. It follows that the transversality condition of the household also holds along any path converging to the steady state

The following proposition summarizes the steady state properties.

Proposition 1. Assume (A1) and (A2). Then a (non-trivial) steady state, $\left(x^{*}, z^{*}, u^{*}\right)$, exists, is unique, and satisfies the transversality condition (20). The steady state is associated with a BGP with the properties:

(i) $g_{Y}^{*}>0, g_{K}^{*}>0$, and $g_{c}^{*}>0$; all three growth rates are increasing functions of the technical change parameters, $\gamma, \beta, \psi$, and $\lambda$, and when learning occurs ( $\beta$ or $\lambda$ positive), also of $n$; 
(ii) $g_{K}^{*} \geq g_{Y}^{*}$ with strict inequality if and only if $\psi>0$ or $\lambda>0$;

(iii) $g_{p}^{*}<0$ when $\psi>0$ or $\lambda>0 ;\left|g_{p}^{*}\right|$ is an increasing function of $\psi$ and $\lambda$; and of $\gamma$ if $\lambda>0$; and of $\beta$ if $\psi>0$ or $\lambda>0$;

(iv) the saving rate in steady state is $s^{*}$ and satisfies $0<s^{*}<\alpha$;

(v) $(1-\alpha) z^{*}<x^{*}<z^{*}$;

(vi) $0<u^{*}<z^{*} /(1+\lambda)$.

Proof. Existence and uniqueness was shown above, provided $s^{*}>0$, which we show in connection with (iv) below. (i) follows immediately from (22), (23), and (26). (ii) was shown above. (iii) follows immediately from (25). (iv) is an application of $s \equiv I / Y=(\dot{K}+\delta K) /(Q Y)=\left(g_{K}+\delta\right) / z$, which follows from $(5)$ and the definition of $z$. In steady state

$$
s=s^{*}=\frac{g_{K}^{*}+\delta}{z^{*}}=\alpha \frac{g_{K}^{*}+\delta}{\theta g_{c}^{*}+\rho+g_{Q}^{*}+\delta}<\alpha \frac{g_{K}^{*}+\delta}{g_{Y}^{*}+g_{Q}^{*}+\delta}=\alpha,
$$

where $g_{Y}^{*}+g_{Q}^{*}=g_{K}^{*}$ follows from constancy of $z$ and the inequality is implied by (A2), which in view of (26) is equivalent to $\theta g_{c}^{*}+\rho>g_{c}^{*}+n=g_{Y}^{*}$. The inequality $s^{*}>0$ in (iv) follows from (i) and $\delta>0$. (v) is implied by (iv) since $s^{*}=1-x^{*} / z^{*}$ and $0<\alpha<1$. The first inequality in (vi) follows from $u^{*}=g_{Y}^{*} / s^{*}$ together with (i) and (iv); in view of (22) and (10) we have $u^{*} / z^{*}=s^{*} u^{*} /\left(s^{*} z^{*}\right)=g_{Y}^{*} /\left(g_{K}^{*}+\delta\right)$ $=\left(g_{K}^{*}-\psi\right) /\left[(1+\lambda)\left(g_{K}^{*}+\delta\right)\right]$, see Appendix A. As $\psi \geq 0$ and $\delta>0$, the second inequality in (vi) follows. We have already shown that $\theta g_{c}^{*}+\rho>g_{Y}^{*}$. This inequality implies, by (28) and constancy of $z \equiv Y /(p K) \equiv Y / V$ in steady state, that $r^{*}$ $>g_{V}^{*}$. The latter inequality ensures that the transversality condition (20) holds in the steady state.

Remark. As long as (A2) holds, all the formulas derived above for growth rates and for $x^{*}, z^{*}, u^{*}, s^{*}$, and $r^{*}$ are valid for any combination of parameter values within the allowed ranges, including the limiting case $\gamma=\beta=\lambda=\psi=n=0$. But in the absence of (A1), that is, when $\gamma=\psi=n=0$, the steady state $\left(x^{*}, z^{*}, u^{*}\right)$ is only an asymptotic steady state. Indeed, it has $0<x^{*}<z^{*}$, but $u^{*}=0$ because, while $Y$ is growing at a diminishing rate, the denominator in $u \equiv Y / J$ goes to infinity 
at a faster speed. So, a viable economy (one with $Y>0$ and $J<\infty$ ) cannot be situated in a steady state with $u^{*}=0$, but it can approach it for $t \rightarrow \infty$ (and will in fact do so when (A2) holds). Thus, when (A1) is not satisfied, the formulas should be interpreted as pertaining to the asymptotic values of the corresponding ratios. And in contrast to (i) of Proposition 1, we get $g_{Y}^{*}=g_{K}^{*}=g_{c}^{*}=0$. This should not be interpreted as if stagnation is the ultimate outcome, however. It is an example of less-than-exponential, but sustained quasi-arithmetic growth (see Groth et al., 2010). Since we are in this paper interested in the speed of convergence to a balanced growth path, we shall concentrate on the case where both (A1) and (A2) hold.

Note that violation of the upper bound on $\lambda$ in (6) implies a growth potential so enormous that a steady state of the system is infeasible and the growth rate of the economy tends to be forever rising. To allow existence of a non-negative $\lambda$ satisfying the parameter inequality in (6) we need $\beta<1$, as was assumed in (2).

\subsection{Transitional dynamics and stability}

We have:

Proposition 2. Assume (A1) and (A2). Let $z_{0}=\bar{z}_{0}$ and $u_{0}=\bar{u}_{0}$, where $\bar{z}_{0}$ and $\bar{u}_{0}$ are given positive numbers. Then there is a neighborhood of $\left(z^{*}, u^{*}\right)$ such that for $\left(\bar{z}_{0}, \bar{u}_{0}\right)$ belonging to this neighborhood, there exists a unique equilibrium path $\left(x_{t}, z_{t}, u_{t}\right)_{t=0}^{\infty}$. The equilibrium path has the property $\left(x_{t}, z_{t}, u_{t}\right) \rightarrow\left(x^{*}, z^{*}, u^{*}\right)$ for $t \rightarrow \infty$.

Proof. In Appendix B it is shown that the Jacobian matrix associated with the dynamic system, evaluated in the steady state, has two eigenvalues with negative real part and one positive eigenvalue. There are two predetermined variables, $z$ and $u$, and one jump variable, $x$. It is shown in Appendix $\mathrm{C}$ that the structure of the Jacobian matrix implies that for $\left(\bar{z}_{0}, \bar{u}_{0}\right)$ belonging to a small neighborhood of $\left(z^{*}, u^{*}\right)$ there always is a unique $x_{0}>0$ such that there exists a solution, $\left(x_{t}, z_{t}, u_{t}\right)_{t=0}^{\infty}$, of the differential equations, (21), (14), and (15), starting from $\left(x_{0}, \bar{z}_{0}, \bar{u}_{0}\right)$ at $t=0$ 
and converging to the steady state for $t \rightarrow \infty$. By (A2) and Proposition 1, the transversality condition (20) holds in the steady state. Hence it also holds along the converging path, which is thus an equilibrium path. All other solution paths consistent with the given initial values, $\bar{z}_{0}$ and $\bar{u}_{0}$, of the state variables diverge from the steady-state point and violate the transversality condition of the household and/or the non-negativity constraint on $K$ for $t \rightarrow \infty$. Hence they can be ruled out as equilibrium paths of the economy.

In brief, the unique steady state is a saddle point and is saddle-point stable.

\subsection{Speed of convergence}

The two eigenvalues with negative real part can be either real or complex conjugate numbers. In our simulations for a broad range of parameter values we never encountered complex eigenvalues. Similarly, the simulations suggested that repeated real negative eigenvalues will never arise for parameter values within a reasonable range. Hence we concentrate on the case of three real distinct eigenvalues two of which are negative. We name the three eigenvalues such that $\eta_{1}<\eta_{2}<0<\eta_{3}$.

Let the vector $\left(x_{t}, z_{t}, u_{t}\right)$ be denoted $\left(x_{1 t}, x_{2 t}, x_{3 t}\right)$. The general formula for the solution to the approximating linear system is $x_{i t}=C_{1 i} e^{\eta_{1} t}+C_{2 i} e^{\eta_{2} t}+C_{3 i} e^{\eta_{3} t}+x_{i}^{*}$, where $C_{1 i}, C_{2 i}$, and $C_{3 i}$ are constants that depend on $\left(x_{10}, x_{20}, x_{30}\right)$. For the equilibrium path of the economy we have $C_{3 i}=0, i=1,2,3$, so that

$$
x_{i t}=C_{1 i} e^{\eta_{1} t}+C_{2 i} e^{\eta_{2} t}+x_{i}^{*}, \quad i=1,2,3
$$

where $C_{1 i}$ and $C_{2 i}$ are constants that depend on the given initial condition $\left(x_{20}, x_{30}\right)$ $=\left(\bar{z}_{0}, \bar{u}_{0}\right)$.

Let the "distance" between the variable $x_{i}, i=1,2,3$, at time $t$ and its steady state value be denoted $\Delta_{i t}$, that is, $\Delta_{i t} \equiv x_{i t}-x_{i}^{*}$. We conceive the speed of convergence of $x_{i}$ as the asymptotic proportionate rate of decline of $\Delta_{i t}$ for $t \rightarrow \infty$. At a 
given $t$ for which $\Delta_{i t} \neq 0$ we have

$$
-\frac{\frac{d \Delta_{i t}}{d t}}{\Delta_{i t}}=\left\{\begin{array}{c}
-\frac{C_{1 i} e^{\eta_{1} t} \eta_{1}+C_{2 i} e^{\eta_{2} t} \eta_{2}}{C_{1 i} e^{\eta_{1} t}+C_{2 i} e^{\eta_{2} t}}=-\frac{\frac{C_{1 i}}{C_{2 i}} e^{\left(\eta_{1}-\eta_{2}\right) t} \eta_{1}+\eta_{2}}{C_{1 i} e^{\left(\eta_{1}-\eta_{2}\right) t}+1}, \quad \text { if } C_{2 i} \neq 0 \\
-\eta_{1}, \quad \text { if } C_{2 i}=0 \text { and } C_{1 i} \neq 0
\end{array}\right.
$$

The two cases appearing here are mutually exclusive and exhaust the possibilities consistent with $x_{i t}-x_{i}^{*} \neq 0$ (see $\left.(29)\right)$.

In view of $\eta_{1}<\eta_{2}<0$, for $C_{2 i} \neq 0$ and for the given $t$ large enough, the absolute value of $\frac{C_{1 i}}{C_{2 i}} e^{\left(\eta_{1}-\eta_{2}\right) t}$ is less than 1 so that $\Delta_{i t} \neq 0$. We see that

$$
\lim _{t \rightarrow \infty}\left(-\frac{\frac{C_{1 i}}{C_{2 i}} e^{\left(\eta_{1}-\eta_{2}\right) t} \eta_{1}+\eta_{2}}{\frac{C_{1 i}}{C_{2 i}} e^{\left(\eta_{1}-\eta_{2}\right) t}+1}\right)=-\eta_{2}
$$

Hence, we define the speed of convergence of $x_{i}$, denoted $\sigma_{i}$, by

$$
\sigma_{i}=\left\{\begin{aligned}
-\eta_{2} & \text { if } C_{2 i} \neq 0 \\
-\eta_{1} & \text { if } C_{2 i}=0 \text { and } C_{1 i} \neq 0
\end{aligned}\right.
$$

When both $C_{1 i}$ and $C_{2 i}$ differ from zero, both negative eigenvalues enter the formula, (29), for the asymptotic solution, but the eigenvalue which is smallest in absolute value, here $\eta_{2}$, is the dominant eigenvalue.

There are two situations to be distinguished: the situation where the dynamic system, (21), (14), and (15), is indecomposable and the situation where it is not. We say the system is indecomposable if all three variables, $x, z$, and $u$, are mutually dependent. On the other hand the system is decomposable if one or two of the three differential equations are decoupled from the remaining part of the system. By inspection of the right-hand sides of (21), (14), and (15), we see that, apart from $s \equiv 1-x / z$, only four parameters enter the coefficients of $x, z$, and $u$, namely $\lambda, \beta, \alpha$, and $\theta$. The values of these parameters govern whether the dynamic system is indecomposable or decomposable. Two parameter value combinations lead to the decomposable situation, namely Case $\mathfrak{D} 1: \lambda=0=\beta, \theta \neq \alpha$; and Case $\mathfrak{D} 2: \lambda=0$, $\beta \geq 0, \theta=\alpha\left(\mathfrak{D}\right.$ for decomposability) ${ }^{9}$

\footnotetext{
${ }^{9}$ In Appendix D the concepts of decomposability and indecomposability are formally defined in terms of properties of the Jacobian matrix associated with the dynamic system.
} 
When learning is operative $(\lambda>0$ or $\beta>0)$, the dynamic system is indecomposable (at least when $\theta \neq \alpha$ ). Consequently the key variables, $x, z$, and $u$, have the same asymptotic speed of convergence. Indeed:

Proposition 3. Assume (A1) and (A2). Let $x_{i 0} \neq x_{i}^{*}, i=1,2,3$. If $\lambda>0$ or $(\beta>0$ and $\theta \neq \alpha$ ), then generically $C_{2 i} \neq 0, i=1,2,3$, and so the same asymptotic speed of convergence, $-\eta_{2}$, applies to all three variables in the dynamic system.

Proof. See Appendix D.

The explanation of this result is that as long as at least part of technical progress is due to learning by investing, the laws of movement for the consumption-capital ratio, $x$, and the output-capital ratio, $z$, are coupled to the law of movement of the sluggish output-experience ratio, $u$ (at least when $\theta \neq \alpha$ ). So the dominant eigenvalue for the $x$ and $z$ dynamics is the same as that for the $u$ dynamics, namely $\eta_{2}$

\subsection{Discontinuity of the speed of convergence for $x$ and $z$ when learning disappears}

When the dynamic system is decomposable, however, the movement of $x$ and $z$ is no longer linked to the slowly adjusting output-experience ratio and therefore, as we shall see, $x$ and $z$ adjust considerably faster. To be specific, consider first the Case $\mathfrak{D} 1$. Here learning by investing is not operative, neither in embodied nor in disembodied form. Then the differential equations for the consumption-capital ratio, $x$, and the output-capital ratio, $z$, are decoupled from the dynamics of the output-experience ratio, $u$. The evolution of $x$ and $z$ is entirely independent of that of $u$ which in turn, however, depends on that of $x$ and $z$. To put it differently, we have a two-dimensional subsystem determining the evolution of $x$ and $z$ and a three-dimensional overall system through which, given the evolution of $x$ and $z$, the differential equation for $u$ determines the evolution of $u$. In any event, $x$ and $z$ are the two variables of primary economic interest, whereas $u$ is of economic interest only to the extent that its movement affects that of $x$ and $z$; in Case $\mathfrak{D} 1$ it does 
not. As $\theta \neq \alpha$, the $(x, z)$ subsystem cannot be decomposed further.

Case $\mathfrak{D} 2$ is the case where, due to the knife-edge condition $\theta=\alpha$, the dynamics of the jump variable $x$ become independent of the dynamics of both state variables, $z$ and $u$, when $\lambda=0$, i.e., when embodied learning is absent. Indeed, with $\theta=\alpha$ and $\lambda=0$, the differential equation for $x$ reduces to $\dot{x}=(x-(\delta+\rho) / \alpha+\delta+n+(1-$ $1 / \alpha) \psi) x$. Then the transversality condition of the household can only be satisfied if $x=x^{*}$ for all $t$. A shift in a parameter affecting $x^{*}$ implies an instantaneous jump of $x$ to the new $x^{*}$. In this case we define the speed of convergence of $x$ as infinite. The state variables $z$ and $u$ will still adjust only sluggishly.

An interesting question is how the speed of convergence of an endogenous variable changes when a parameter value changes so that the system shifts from being indecomposable to being decomposable. To spell this out we need more notation. Consider again Case $\mathfrak{D} 1$ where $\theta \neq \alpha$ and learning of any form is absent. We let the eigenvalues associated with the subsystem for $x$ and $z$ in this case be $\eta_{1}=\bar{\eta}_{1}$ and $\eta_{3}=\bar{\eta}_{3}$, where $\bar{\eta}_{1}<0<\bar{\eta}_{3}$. The third eigenvalue, $\eta_{2}$, belongs to the total system but does not in this case influence the $x$ and $z$ dynamics; it is denoted $\bar{\eta}_{2}$ and turns out to equal $-g_{Y}^{*}<0$ (see Appendix E). In the sub-case of $\mathfrak{D} 2$ where $\beta=0$ in addition to $\theta=\alpha$ and $\lambda=0$, we let the values taken by the eigenvalues be denoted $\tilde{\eta}_{1}, \tilde{\eta}_{2}$, and $\tilde{\eta}_{3}$

For realistic parameter values, $\bar{\eta}_{2}$ and $\tilde{\eta}_{2}$ are smaller in absolute value than $\bar{\eta}_{1}$ and $\tilde{\eta}_{1}$, respectively. That is, from an empirical point of view we can assume $\bar{\eta}_{1}$ $<\bar{\eta}_{2}<0<\bar{\eta}_{3}$ as well as $\tilde{\eta}_{1}<\tilde{\eta}_{2}<0<\tilde{\eta}_{3}$. Given these inequalities, the speed of convergence of one or more of the variables changes discontinuously as learning, whether embodied or disembodied, tends to vanish:

Proposition 4. Assume (A1) and (A2). Let $\bar{\eta}_{1}<\bar{\eta}_{2}<0<\bar{\eta}_{3}$ and $\tilde{\eta}_{1}<\tilde{\eta}_{2}<0$ $<\tilde{\eta}_{3}$. We have:

(i) If $\theta \neq \alpha$, then, for $(\beta, \lambda) \rightarrow(0,0)^{+}$, in the limit where learning disappears, an upward switch occurs in the speed of convergence for $x$ and $z$ from the value $-\bar{\eta}_{2}$ to $-\bar{\eta}_{1}$. 
(ii) If $\theta=\alpha, \beta=0$, and $\lambda>0$, then, for $\lambda \rightarrow 0^{+}$, in the limit where learning disappears, two upward switches occur. The speed of convergence for $x$ shifts from the value $-\tilde{\eta}_{2}$ to infinity. And the speed of convergence for $z$ shifts from the value $-\tilde{\eta}_{2}$ to $-\tilde{\eta}_{1}>-\tilde{\eta}_{2}$.

(iii) If $\theta=\alpha, \lambda=0$, and $\beta \geq 0$, the speed of convergence for $x$ is always infinite. But for $\beta \rightarrow 0^{+}$, in the limit where learning disappears, the speed of convergence for $z$ switches from the value $-\tilde{\eta}_{2}$ to $-\tilde{\eta}_{1}>-\tilde{\eta}_{2}$.

Proof. See Appendix E.

Result (i) is the generic result on which our numerical calculations concentrate. The intuition behind result (i) is that as long as at least a part of technical progress is due to learning by investing (either $\lambda$ or $\beta$ positive), the laws of movement for $x$ and $z$ are generically coupled to the law of movement of the sluggish outputexperience ratio, $u$. But if learning by investing disappears, the movement of $x$ and $z$ is no longer hampered by this slow-adjusting factor and therefore $x$ and $z$ adjust much faster. In for instance Figure 1 below, for $\beta=\psi=0$ and with the baseline parameter combination indicated in Table 2 below, this discontinuity in the limit shows up as a jump in the convergence speed for $x$ and $z$ from 0.03 to above 0.08 when $\lambda \rightarrow 0^{+}$.

The intuition behind result (ii) is similar, except that here the dynamics become fully recursive in the limit. This has two interesting implications. First, the jump variable, $x$, ceases to be influenced by the movement of the state variables, $z$ and $u$, and can therefore adjust with infinite speed. Second, $z$ ceases to be influenced by the slow-adjusting $u$. Result (iii) refers to a situation where the speed of convergence of the jump variable $x$ is infinite even for $\beta>0$ (that is, when disembodied learning is present) and remains so in the limit for $\beta \rightarrow 0^{+}$. Moreover, in the limit $z$ ceases to be influenced by the slow-adjusting $u$ and so the speed of convergence of $z$ jumps.

Most empirical evidence suggests $\theta \geq 1>\alpha$. So the results (ii) and (iii), relying on the knife-edge case $\theta=\alpha$, are of limited interest. On the other hand, this case allows an explicit solution for the time path of one or more of the variables. 
Therefore at several occasions this case has received attention in the literature, for example in connection with the Lucas (1988) human capital accumulation model (see Xie (1994) and Boucekkine and Ruiz-Tamarit (2004)).

For mathematical convenience this section has talked about limiting values of the speed of convergence for the two forms of learning approaching zero. We may turn the viewpoint round and end this section with the conclusion that as soon as learning from gross investment becomes positive, and thereby part of the growth engine, the asymptotic speed of convergence displays a discrete fall.

\section{Alternative model: Learning from net invest- ment}

The benchmark model above assumes that learning stems from gross investment. What difference does it make if instead the basis of learning, whether embodied or disembodied, is net investment? To provide an answer, we now describe the case where it is the experience originating in cumulative net investment that drives productivity. This case seems less plausible, since presumably the total amount of newly produced equipment provides new stimuli and experience from which to learn, whatever the depreciation on existing equipment. Yet it is certainly the more popular case in the literature, probably because of its mathematical simplicity. ${ }^{10}$

We replace (3) by $J_{t}=\int_{-\infty}^{t} I_{\tau}^{n} d \tau$, where $I_{\tau}^{n}$ denotes net investment, $I_{\tau}-\delta K_{\tau}$, at time $\tau$. Then $\dot{K}_{\tau}=I_{\tau}^{n}$ and by integration $J_{t}=K_{t}$. Hence (11) and (12) become $g_{A}$ $=\beta g_{K}+\gamma$ and $g_{Q}=\lambda g_{K}+\psi$, respectively. To avoid growth explosion, we need that $\lambda$ satisfies $0 \leq \lambda<(1-\alpha)(1-\beta)$, which is sharper than the restriction in (6). Since $J$ is no longer distinct from $K$, the dynamic system reduces to two dimensions:

$$
\begin{aligned}
& g_{x}=\frac{1}{\theta}(\alpha z-\delta-\rho)+\left[\left(1-\frac{1}{\theta}\right) \lambda-1\right](z-x-\delta)+n+\left(1-\frac{1}{\theta}\right) \psi \\
& g_{z}=[\lambda-(1-\alpha)(1-\beta)](z-x-\delta)+(1-\alpha)(\gamma+n)+\psi
\end{aligned}
$$

\footnotetext{
${ }^{10}$ Leading textbooks such as Acemoglu (2009), Aghion and Howitt (1998, 2009), Barro and Sala-i-Martin (2004), de la Croix and Michel (2002), and Valdez (1999), concentrate on this case and predominantly on learning in the disembodied form.
} 
where, as before, $x \equiv C /(p K)$ and $z \equiv Y /(p K)$.

Also this simpler model has a unique saddle-point stable steady state (see Appendix F). The long-run growth rate of per capita consumption is

$$
g_{c}^{*}=\frac{(1-\lambda)(1-\alpha) \gamma+[\alpha+(1-\alpha) \beta] \psi+[(1-\alpha) \beta+\alpha \lambda] n}{(1-\alpha)(1-\beta)-\lambda} .
$$

To ensure that the discounted utility integral is bounded and the transversality condition satisfied, we need that $\rho-n>(1-\theta) g_{c}^{*}$. We assume the parameter values are such that this inequality is fulfilled.

Again, the relative price of capital equipment is falling if there is embodied technical progress. Indeed,

$$
g_{p}^{*}=-g_{Q}^{*}=-\frac{(1-\alpha)[(1-\beta) \psi+\lambda(\gamma+n)]}{(1-\alpha)(1-\beta)-\lambda}<0,
$$

if $\psi>0$ or $\lambda>0$. Embodied technical progress leads also to a falling $Y / K$ such that ultimately the output-capital ratio in value terms, $Y /(p K) \equiv z$, stays constant.

We note in passing that this model subsumes several models in the literature as special cases:

1. The simple neoclassical growth model: $\gamma>0, \beta=\lambda=\psi=0$.

2. Arrow-Romer model, the "Arrow version": $0<\beta<1, \gamma=\lambda=\psi=0$.

3. Arrow-Romer model, the "Romer version": $\beta=1, n=\gamma=\lambda=\psi=0$.

4. Jovanovic and Rousseau (2002): $0<\lambda<1-\alpha, \delta=\gamma=\beta=\psi=\theta=0 .{ }^{11}$

5. Boucekkine et al. (2003): knife-edge link between $\lambda$ and $\beta$ : $\lambda=(1-\alpha)(1-$ $\beta), \gamma=\psi=0 .^{12}$

\footnotetext{
${ }^{11}$ The linear utility assumption, $\theta=0$, implies $r=\rho$ in equilibrium. On the other hand, the authors extend the model by incorporating a second capital good (like structures), not taking part in the embodied learning. And it is only in the theoretical analysis that the simplifying assumption that learning comes from net investment is relied upon.

${ }^{12}$ Strictly speaking, this description of Boucekkine et al. (2003) only covers the case $n=0$. By letting the learning effects come from net investment per capita, the authors can allow $n>0$ without growth explosion, unlike the "Romer version" above.
} 
Number 2 and 3 in the list are the standard textbook models of learning by investing referred to in the first paragraph of Section 2. The original contributions in Arrow (1962) and Romer (1986) are more sophisticated than these popular cases from textbooks. Discussing these aspects would take us too far, however.

We now return to the general version of the net-investment based learning model, summarized in (31) and (32). The case $\theta>\alpha$ is the empirically plausible case to be considered in the numerical simulations below. In this case (in fact whenever $\theta \neq \alpha)$ the dynamic system is indecomposable even for $\lambda=0$. The absolute value of the unique negative eigenvalue is the common speed of convergence for $x$ and $z$.

Contrary to the benchmark model of the preceding sections, this model version exhibits no discontinuity in the speed of convergence in the limit as $(\beta, \lambda) \rightarrow(0,0)^{+}$, i.e., as learning disappears. Indeed, when learning originates in net investment, the variable that drives productivity is cumulative net investment and thereby simply the capital stock. The dynamics of the capital stock is part of the dynamics of $x$ and $z$ whether or not any learning parameter is positive. It is otherwise in the benchmark model where as soon as a learning parameter becomes positive, the dynamics of $x$ and $z$ is coupled to the dynamics of an entirely new variable, cumulative gross investment. In the limiting case of $\beta=\lambda=0$, i.e., no learning, the two models are of course identical.

We are now ready to consider numerical results for the benchmark model of the preceding sections as well as the present simpler, alternative model version.

\section{Results from simulations}

Proposition 4 implies the qualitative result that as soon as learning from gross investment becomes part of the growth engine, the asymptotic speed of convergence (from now abbreviated SOC) of $x$ and $z$ drops. Considering reasonable calibrations, four main quantitative questions suggest themselves. First, by how much does the introduction of learning lower SOC? Second, if more weight is put on learning and less weight on unspecified exogenous sources of technical progress, by how much is 
SOC affected? Third, how much does it matter whether learning is based on gross or net investment? Fourth, does embodiment of technical change matter for SOC? Numerical simulations, addressing these questions, are presented in the following.

What we call baseline values of the background parameters are listed in Table 2. Tables and graphs below are based on these baseline values which may be considered standard and noncontroversial. Appendix G contains sensitivity analysis, in particular with respect to the value of $\theta$, since this parameter affects SOC considerably.

TABLE 2

\section{BASELINE VALUES OF BACKGROUND PARAMETERS}

\begin{tabular}{ll}
\hline \hline Preference parameters & $\rho=0.02, \theta=1.75$ \\
Production parameters & $\alpha=0.324, \delta=0.05$ \\
Population growth & $n=0.01$
\end{tabular}

Note. The time unit is one year.

The parameters of primary interest are the technical change parameters: $\beta, \gamma, \lambda$, and $\psi$. The empirical literature does not provide firm conclusions as to the relative importance of learning by investing (including learning spillovers) versus other sources of long-run growth and the relative importance of embodied learning vs. disembodied learning. To clarify the potential quantitative role of these parameters for SOC, we vary them in pairs in the simulations so as to hold constant the growth rate of per capita consumption. Specifically, if one technical change parameter is increased, another technical change parameter is decreased so as to ensure $g_{c}^{*}=0.02$. In this way we can study the role of the composition of technical progress without interference from the size of the growth rate.

\subsection{The role of embodied learning}

Panel A of Table 3 presents major results for the case where the strength, $\lambda$, of embodied learning vis-a-vis the strength, $\gamma$, of disembodied exogenous progress is in focus (at the same time as $\beta=\psi=0$ ). The baseline combination of $\lambda$ and $\gamma$ 
appears in the second row. With this combination together with the baseline values of the background parameters, cf. Table 2, important stylized facts for a modern industrialized economy are reproduced by the model. Per capita consumption grows at a rate of $2 \%$ per year, $26 \%$ of output is devoted to investment, ${ }^{13}$ and the outputcapital ratio is 0.40 . Moreover, embodied technical change accounts for $60 \%$ of the growth in per capita output, leaving the remaining $40 \%$ as due to disembodied technical change $\left(\gamma / g_{c}^{*}=0.4\right)$. This corresponds to the estimates by Greenwood et al. (1997). With $g_{p}^{*}=-0.03$ the baseline case roughly captures the observation that the relative price of capital equipment vis-a-vis consumption goods has in the US declined at a yearly rate of 3\% in the period 1950-1990 (Greenwood et al. 1997). ${ }^{14}$ The asymptotic speed of convergence amounts to about $1.6 \%$ per year, which corresponds to estimates in the seminal studies by Mankiw et al. (1992) and Barro and Sala-i-Martin (2004).

Comparing the rows in Panel A of Table 3 we see the impact of raising embodied learning as a source of technical change while lowering disembodied exogenous technical change so as to hold constant the per capita consumption growth rate at $2 \%$ per year. Thus for small $\lambda$ the main source of technical progress is disembodied exogenous technical change, while for large $\lambda$ it is embodied learning from gross investment.

Several features are worth mentioning. First, if $\lambda=0$ (the standard neoclassical growth model), the asymptotic speed of convergence for $x$ and $z$ equals $8.78 \%$, a value which is considered much too high by Mankiw et al. (1992) and Barro and Sala-i-Martin (2004). With the indicated baseline value of $\lambda$, however, the asymptotic speed of convergence takes on a significantly lower value of around $2 \%$. To obtain a SOC at this level, the standard neoclassical growth model requires

\footnotetext{
${ }^{13}$ When taking investment in consumer durables into account in addition to fixed capital investment, an investment share of GDP of around one fourth is empirically realistic.

${ }^{14}$ We only say "roughly captures" because in our model, $p$ is the relative price of an aggregate capital good, whereas the 3\% from Greenwood et al. (1997) excludes structures from the price index. On the other hand, studies by Jovanovic and Rousseau (2002) and Sakellaris and Wilson (2004) suggest a speed up of the fall in the relative price of capital equipment due to the expanding role of computers and IT-related technology.
} 
an output elasticity with respect to capital as high as $\alpha=0.75$ (interpreted as reflecting the productive role of an expanded measure of capital including human capital, cf. Barro and Sala-i-Martin, 2004, p. 110). Table 3 shows that with embodied learning from investment accounting for $60 \%$ of the growth in per capita output (or consumption), the empirically endorsed SOC of around $2 \%$ is obtained without requiring the output elasticity with respect to capital to exceed one third.

TABLE 3

SPEED OF CONVERGENCE AS THE EMBODIED LEARNING PARAMETER RISES

\begin{tabular}{|c|c|c|c|c|c|c|c|c|c|c|}
\hline & $\lambda$ & $\gamma$ & $g_{c}^{*}$ & $r^{*}$ & $s^{*}$ & $(Y /(p K))^{*}$ & $g_{p}^{*}$ & $\gamma / g_{c}^{*}$ & $\sigma_{x}, \sigma_{z}$ & $\sigma_{u}$ \\
\hline \multicolumn{11}{|c|}{ PANEL A. Adjustment of $\gamma$ such that $g_{c}^{*}=0.02$} \\
\hline & 0.000 & 0.020 & 0.02 & 0.055 & 0.25 & 0.32 & -0.00 & 1.00 & 8.77 & 3.00 \\
\hline \multirow[t]{2}{*}{ Baseline } & 0.833 & 0.008 & 0.02 & 0.055 & 0.26 & 0.40 & -0.03 & 0.40 & 1.57 & 1.57 \\
\hline & 1.389 & 0.000 & 0.02 & 0.055 & 0.27 & 0.45 & -0.04 & 0.00 & 0.80 & 0.80 \\
\hline \multicolumn{11}{|c|}{ PANEL B. No adjustment of $\gamma$} \\
\hline & 0.000 & 0.020 & 0.02 & 0.055 & 0.25 & 0.32 & -0.00 & 1.00 & 8.77 & 3.00 \\
\hline & 0.833 & 0.020 & 0.04 & 0.090 & 0.25 & 0.56 & -0.04 & 0.50 & 2.60 & 2.60 \\
\hline & 1.389 & 0.020 & 0.08 & 0.160 & 0.26 & 1.03 & -0.13 & 0.25 & 2.33 & 2.33 \\
\hline \multicolumn{11}{|c|}{ PANEL C. Adjustment of $\gamma$ such that $g_{c}^{*}=0.02 ;$ learning from $I^{n}$} \\
\hline & 0.000 & 0.020 & 0.02 & 0.055 & 0.25 & 0.32 & -0.00 & 1.00 & 8.77 & - \\
\hline & 0.455 & 0.008 & 0.02 & 0.055 & 0.26 & 0.40 & -0.03 & 0.40 & 2.75 & - \\
\hline & 0.581 & 0.000 & 0.02 & 0.055 & 0.27 & 0.45 & -0.04 & 0.00 & 1.20 & - \\
\hline
\end{tabular}

Notes. Baseline values of background parameters as given in Table $2 ; \beta=0, \psi=0 ; \sigma_{i}$ shown in percentage points. Panels A and B: embodied learning from gross investment; Panel C: embodied learning from net investment.

Second, the impact of raising embodied learning further while lowering disembodied exogenous technical change results in a still lower speed of convergence. The explanation is that a higher relative weight to learning in the "growth engine" means a higher relative weight to the slow-adjusting cumulative investment experience that feeds learning.

The reason that we adjust $\gamma$ downwards when raising $\lambda$ is that otherwise the values of several key variables would not remain within ranges that seem empirically relevant (from a historical perspective). To document this, Panel B of Table 3 leaves 
$\gamma$ fixed. The result is that the growth rate of per capita consumption rises to $8 \%$; the rate of interest rises to $16 \%$; and the output-capital ratio rises to a value above 1. Since such values are far away from what we have observed in the data, the associated speeds of convergence (higher than in Panel A) are of limited interest. Of course, here we take a backward-looking perspective. It is possible that the shift to a higher $\lambda$ that seems associated with the computer revolution will result in higher future per capita growth, as conjectured by, e.g., Jovanovic and Rousseau $(2002) \cdot{ }^{15}$

In Panel $\mathrm{C}$ of Table 3 learning stems from net investment rather than gross investment as in the model of Section 4. The second row of Panel C shows that for $\lambda=0.455$ this model reproduces the same magnitudes of key endogenous variables as the baseline row in Panel A. But along with a rise in the fraction of the given $g_{c}^{*}$ that is due to embodied learning stemming from net investment, SOC declines less strongly than in Panel A.

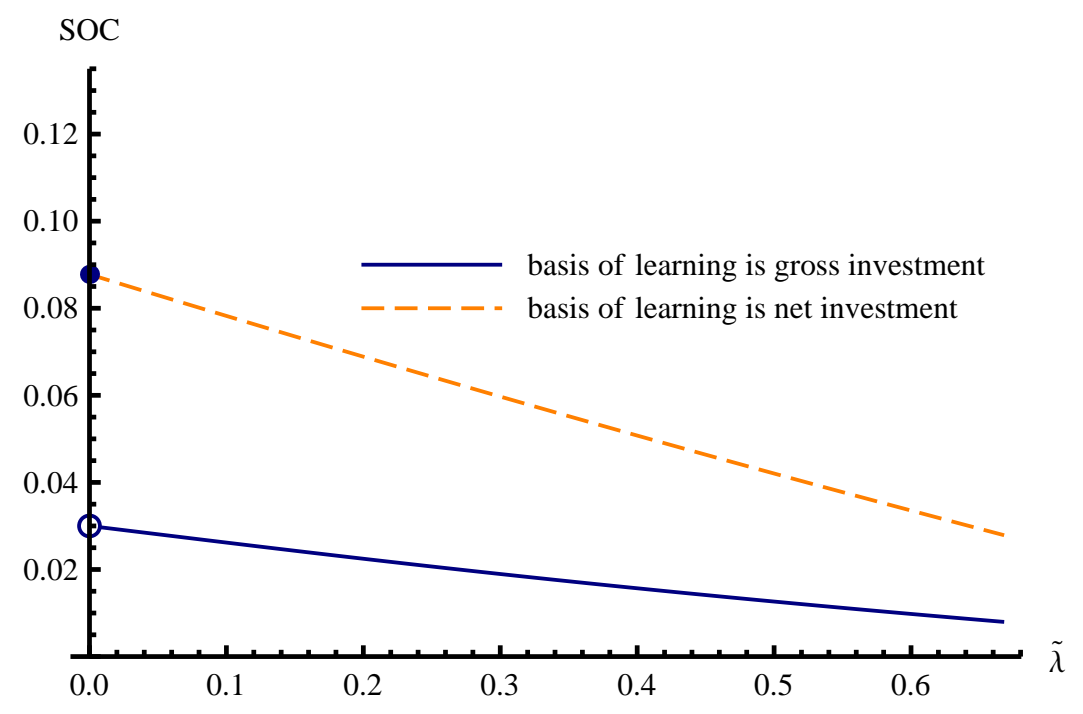

Figure 1: Speed of convergence as the normalized embodied learning parameter, $\tilde{\lambda}$, rises and $\gamma$ is adjusted so as to maintain $g_{c}^{*}=0.02$. Note: $\beta=0, \psi=0 ; \alpha=0.324$.

\footnotetext{
${ }^{15}$ The last row in Panel $\mathrm{B}$, including the sizeable $-g_{p}^{*}$, is not far from the (informal) forecast of growth "in the coming decades" suggested by Jovanovic and Rousseau (2002). For the case of linear utility (i.e., $\theta=0$ ) and $\gamma=\beta=\psi$, Jovanovic and Rousseau derive an explicit formula showing SOC to be decreasing in $\beta$. But since the authors do not adjust any other parameter, also growth is rising in the exercise.
} 
This feature is displayed in more detail in Figure 1. The solid curve shows $\mathrm{SOC}^{16}$ when the basis of learning is gross investment. At a significantly higher position is the dashed curve which shows SOC when the basis of learning is net investment. The variable along the horizontal axis, named $\tilde{\lambda}$, is the learning parameter normalized so as to ensure a common support, i.e., $\tilde{\lambda} \in[0,1]$, for the two cases. Specifically, $\tilde{\lambda} \equiv \lambda \alpha /[(1-\alpha)(1-\beta)]$ when learning is based on gross investment; and $\tilde{\lambda} \equiv$ $\lambda /[(1-\alpha)(1-\beta)]$ when learning is based on net investment. The range for $\tilde{\lambda}$ shown in the figure does not go beyond 0.67 because higher values would require a negative value of $\gamma$ to maintain $g_{c}^{*}=0.02$.

The intuition behind that SOC is lower when the basis of learning is gross investment than when it is net investment, is that the former basis involves more overhang from the past. Thereby the transitional dynamics becomes more sluggish.

Figure 1 also displays the interesting discontinuity in SOC for $x$ and $z$ as learning from gross investment becomes positive. This discontinuity, drawn attention to in Proposition 4, appears as a drop from the solid bullet on the vertical axis in Figure 1 to the hollow bullet. The solid bullet is situated where the dashed curve hits the vertical axis. This is because the two models are identical in the special case of no learning. As we already know from Section 4, when the learning parameter in the "net-investment framework" shifts from nil to positive, no discontinuity in SOC arises. In contrast, in the "gross-investment framework" such a shift couples the dynamics of $x$ and $z$ to that of a variable not involved before, namely the slow-adjusting cumulative gross investment.

Figure 2 is analogue to Figure 1 except that it is the embodied exogenous technical change parameter, $\psi$, that is adjusted when the normalized embodied learning parameter rises (while $\gamma=\beta=0$ ). The resulting pattern is rather similar to that in Figure 1. SOC is quite sensitive to the fraction of embodied productivity increases coming from learning rather than from unspecified exogenous factors. And the vertical distance between the two curves is again substantial, in fact even larger

\footnotetext{
${ }^{16}$ From now on, in figures as well as text, "SOC" refers to SOC of $x$ and $z$.
} 


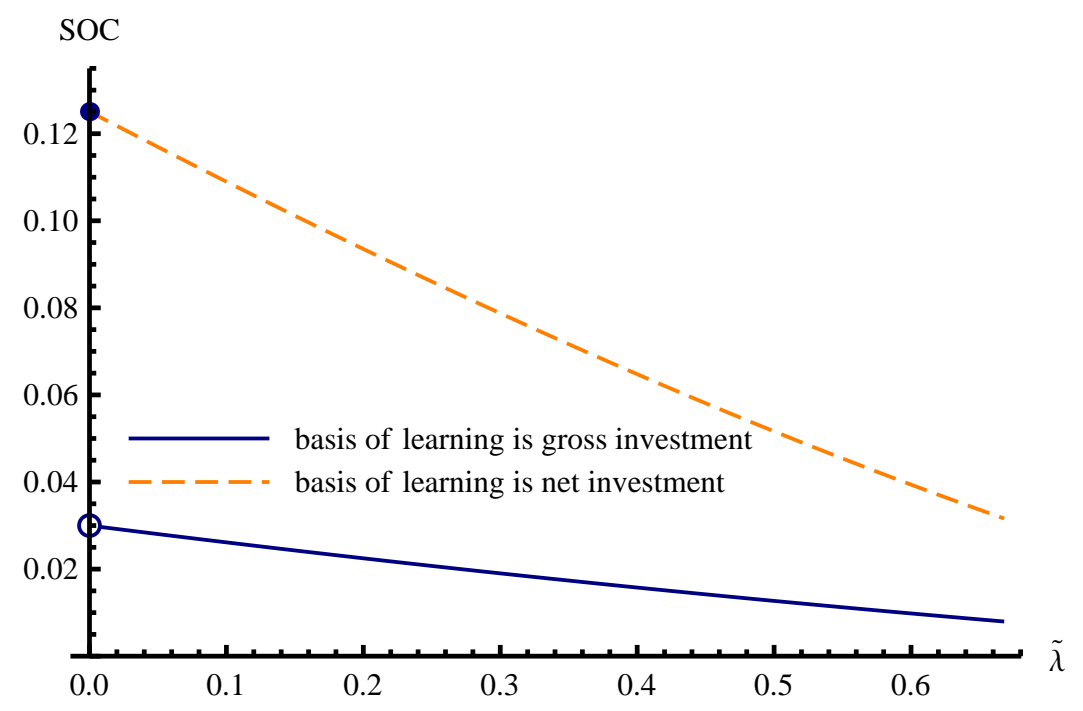

Figure 2: Speed of convergence as the normalized embodied learning parameter, $\tilde{\lambda}$, rises and $\psi$ is adjusted so as to maintain $g_{c}^{*}=0.02$. Note: $\beta=0, \gamma=0 ; \alpha=0.324$.

than before. That is, when a combination of embodied learning and embodied exogenous technical change drives productivity increases, SOC is very sensitive to whether learning is based on net or gross investment.

\subsection{The role of disembodied learning}

Although, for example, Greenwood et al. (1997) found that disembodied technical change accounts for only about $40 \%$ of the growth in output per hours worked, still the impact of whether its source is learning or exogenous, i.e., originating in factors outside the model, is of interest. Figure 3 shows how SOC changes as the strength, $\beta$, of disembodied learning is raised at the same time as disembodied exogenous technical change is lowered so as to hold constant $g_{c}^{*}$ (while $\lambda=\psi=0$ ). ${ }^{17}$ The pattern is quite similar to that in Figure 1 for the embodied learning case: a) a rise in the fraction of disembodied technical change coming from learning rather than being exogenous lowers SOC; b) there is a substantial drop in SOC for $x$ and $z$ as learning from gross investment becomes positive; and c) going from the stippled "net-investment curve" to the solid "gross-investment curve" entails more than a

\footnotetext{
${ }^{17}$ Again the range of the abscissa is limited to values not requiring the adjusting variable to take on a negative value to maintain $g_{c}^{*}=0.02$. This principle is also followed in the ensuing figures.
} 
halving of SOC.

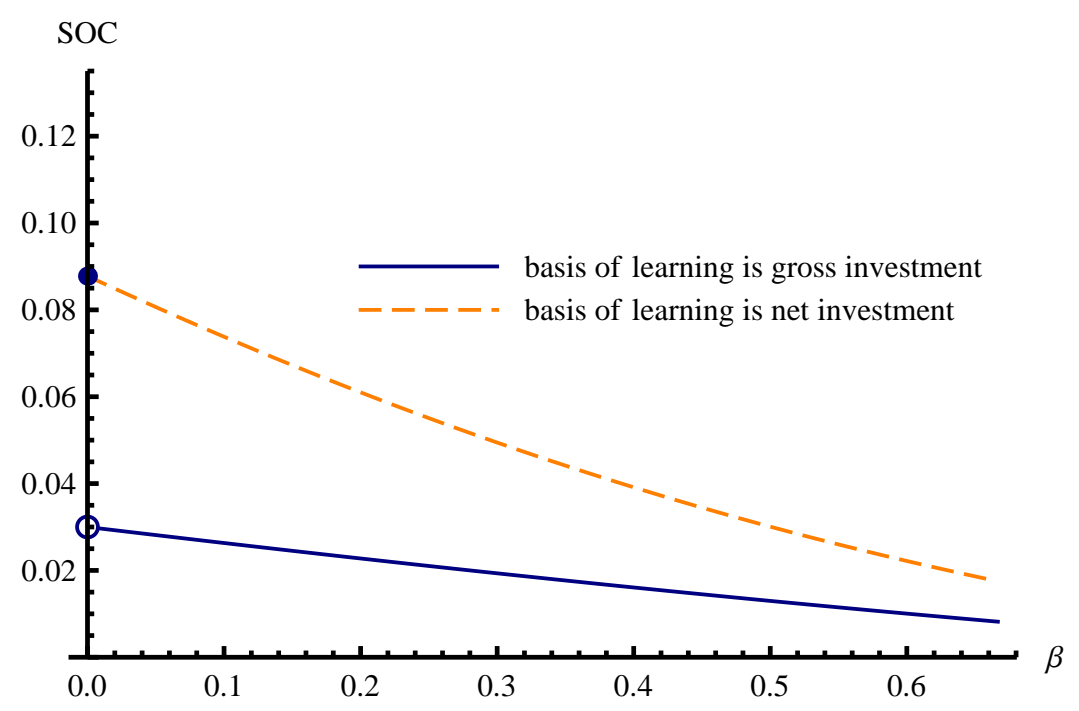

Figure 3: Speed of convergence as the disembodied learning parameter, $\beta$, rises and $\gamma$ is adjusted so as to maintain $g_{c}^{*}=0.02$. Note: $\lambda=0, \psi=0 ; \alpha=0.324$.

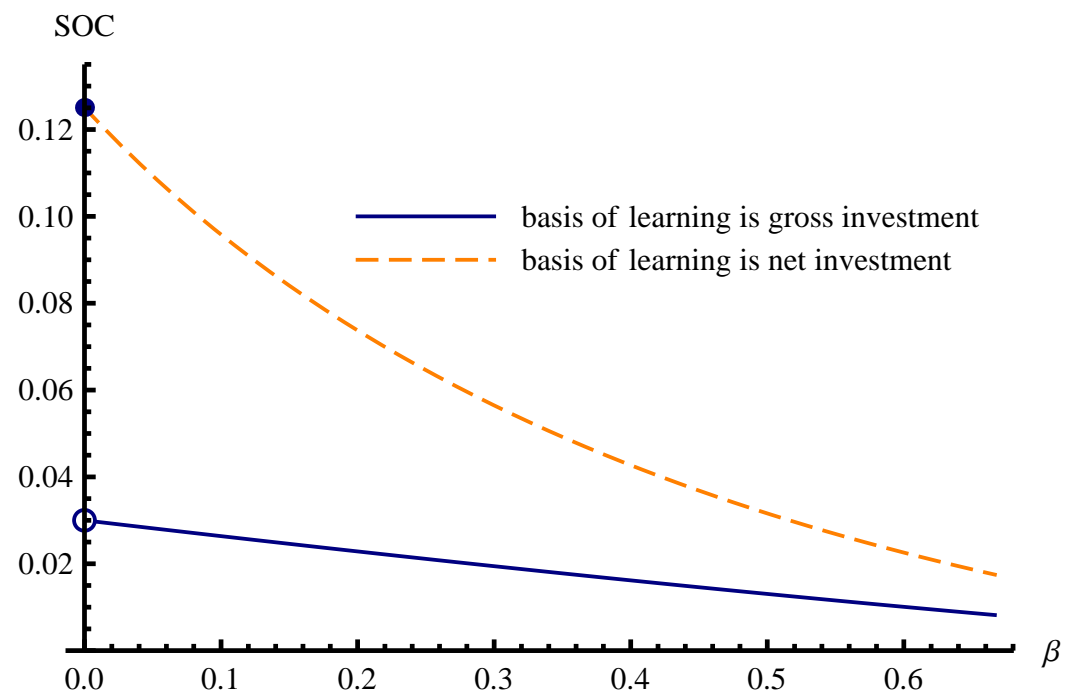

Figure 4: Speed of convergence as the disembodied learning parameter, $\beta$, rises and $\psi$ is adjusted so as to maintain $g_{c}^{*}=0.02$. Note: $\lambda=0, \gamma=0 ; \alpha=0.324$.

In Figure 4 it is instead the strength, $\psi$, of embodied exogenous technical change that is adjusted as $\beta$ rises (while $\gamma=\lambda=0$ ). Again we see: a) a falling SOC; b) a significant discontinuity as learning becomes operative; and c) a persistent difference in the level of the two curves. 
The overall conclusion from this and the previous subsection is that the source of technical change and the basis of learning matter a lot for SOC and does so whether technical change is of embodied or disembodied form.

\subsection{The role of embodiment as such}

Empirical studies by, e.g., Jovanovic and Rousseau (2002) and Sakellaris and Wilson (2004) find that ICT technologies result in faster decline in the relative price of capital equipment vis-a-vis consumption goods than earlier technology revolutions. This can be seen as reflecting a rising tendency for technical change to take the embodied form. ${ }^{18}$

Is such a tendency likely to result in a higher speed of convergence for the economy? Earlier theoretical literature leads to the presumption that the answer might be a yes. At least for Solow-style models with a constant saving rate, Phelps (1962) and Williams and Crouch (1972) thus showed that when a higher fraction of exogenous productivity increases are embodied rather than disembodied, a higher SOC appears.

By disentangling the impact of the form of technical progress from that of its source, let us check whether embodiment generally has such an effect. Figure 5, where all technical progress is exogenous, confirms the supposition from the early literature. SOC is seen to be an increasing function of the fraction of the exogenous productivity increases that are embodied. (As there is no learning, the usual distinction as to the basis of learning is irrelevant and only one curve appears in Figure 5.)

Interestingly, when the source of technical progress is instead learning, embodiment does not increase SOC. In Figure 6 all technical change is due to learning. Not only does this generate a very low SOC but SOC is essentially independent of

\footnotetext{
${ }^{18}$ Tables A, D, E, and $\mathrm{F}$ in the appendix show that $g_{p}^{*}$ is quite sensitive to a rise in the fraction of technical change that is embodied. On the other hand, if embodied exogenous technical change, $\psi$, is the adjusting parameter when embodied learning rises (Table B), $g_{p}^{*}$ is unaffected (but high since all technical change is in this case embodied). Indeed, the constancy of $g_{p}^{*}$ in this case follows analytically from the formula (25) with $\gamma=\beta=0$ and $\psi$ as a function of $\lambda$ so that $g_{c}^{*}=0.02$.
} 


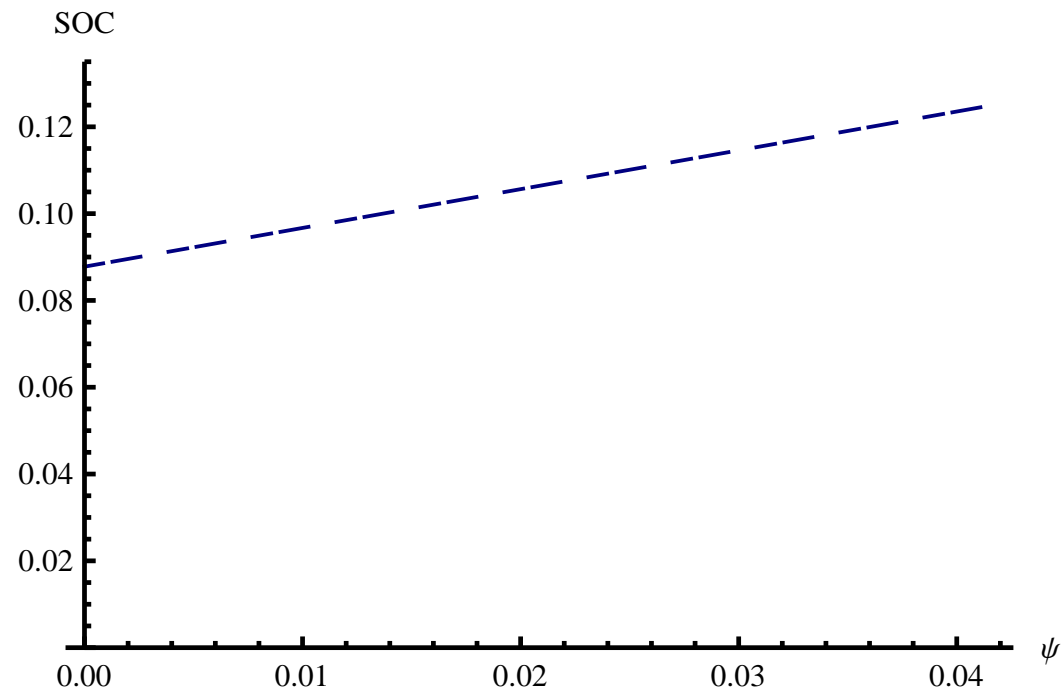

Figure 5: Speed of convergence as the embodied exogenous change parameter, $\psi$, rises and $\gamma$ is adjusted so as to maintain $g_{c}^{*}=0.02$. Note: $\lambda=0, \beta=0 ; \alpha=0.324$.

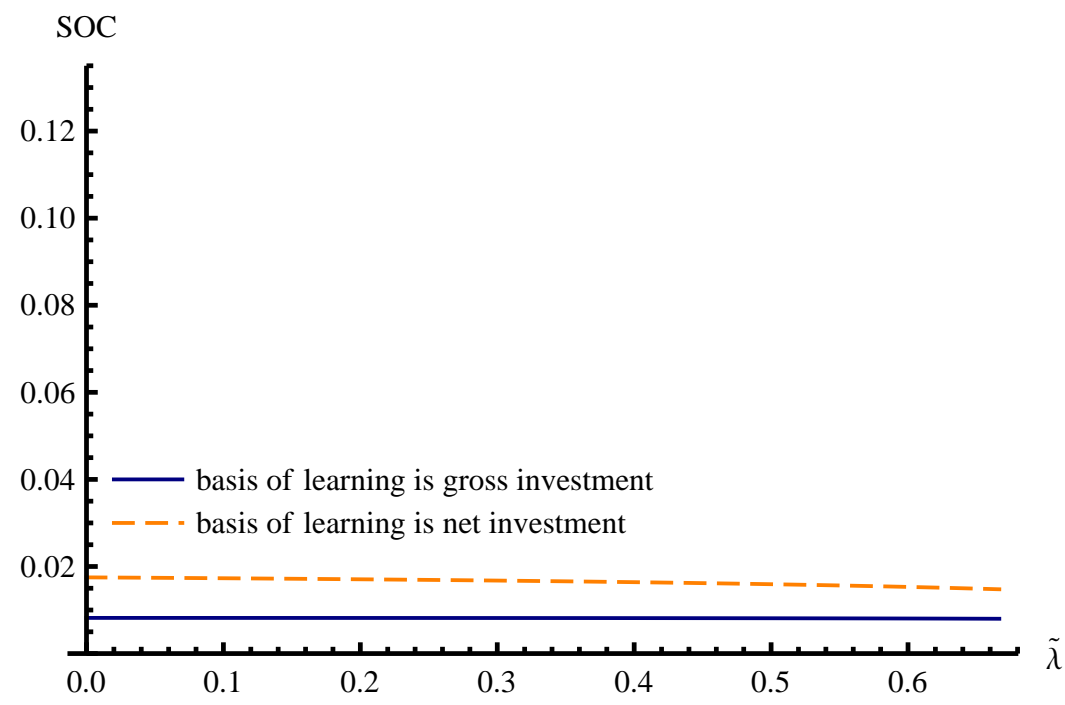

Figure 6: Speed of convergence as the normalized embodied learning parameter, $\tilde{\lambda}$, rises and $\beta$ is adjusted so as to maintain $g_{c}^{*}=0.02$. Note: $\gamma=0, \psi=0 ; \alpha=0.324$. 
the fraction of the learning taking the embodied form rather than the disembodied form.Thus, the form of learning has in this case essentially no bearing on SOC. ${ }^{19}$

We conclude that a rising degree of embodiment of technical change in the wake of the computer revolution does not seem likely to bring about a rising SOC, at least not as long as the overall productivity growth rate remains non-increasing.

\subsection{Other aspects}

It is well-known that a rise in the output elasticity with respect to capital, everything else equal, tends to decrease the speed of convergence. If a disturbance raises the output-capital ratio and interest rate temporarily above steady state level and therefore induces fast capital accumulation, a higher output elasticity with respect to capital means that the output-capital ratio and interest rate are less sensitive to the capital intensity. Therefore the adjustment is slowed down by a high output elasticity with respect to capital.

When the basis of learning is net investment, the effective output elasticity with respect to capital is $\alpha+(1-\alpha) \beta$ rather than just $\alpha$. This raises the question whether the negative slope of the stippled curve in for example Figure 3 is due to the capital-elasticity effect of a rising $\beta$ on the effective output elasticity with respect to capital rather than to the learning effect. The stippled curve in Figure 7 shows that the answer is affirmative: along with the rising $\beta$, we here adjust not only $\gamma$ so as to maintain $g_{c}^{*}=0.02$, but also $\alpha$ so as to maintain $\alpha+(1-\alpha) \beta=0.5$; as a result SOC is more or less constant, in fact slightly increasing. When the basis of learning is gross investment, however, a similar adjustment of $\alpha$ does not change the pattern qualitatively, but makes the slope less steep (compare the solid curve in Figure 7 with that in Figure 3). ${ }^{20}$

It is also well-known that the speed of convergence in a growth model generally tends to slow down as the desire for consumption smoothing, $\theta$, rises and the pop-

\footnotetext{
${ }^{19}$ There is, however, the usual level difference depending on whether learning stems from gross or net investment.

${ }^{20}$ See also Table C in Appendix G.
} 


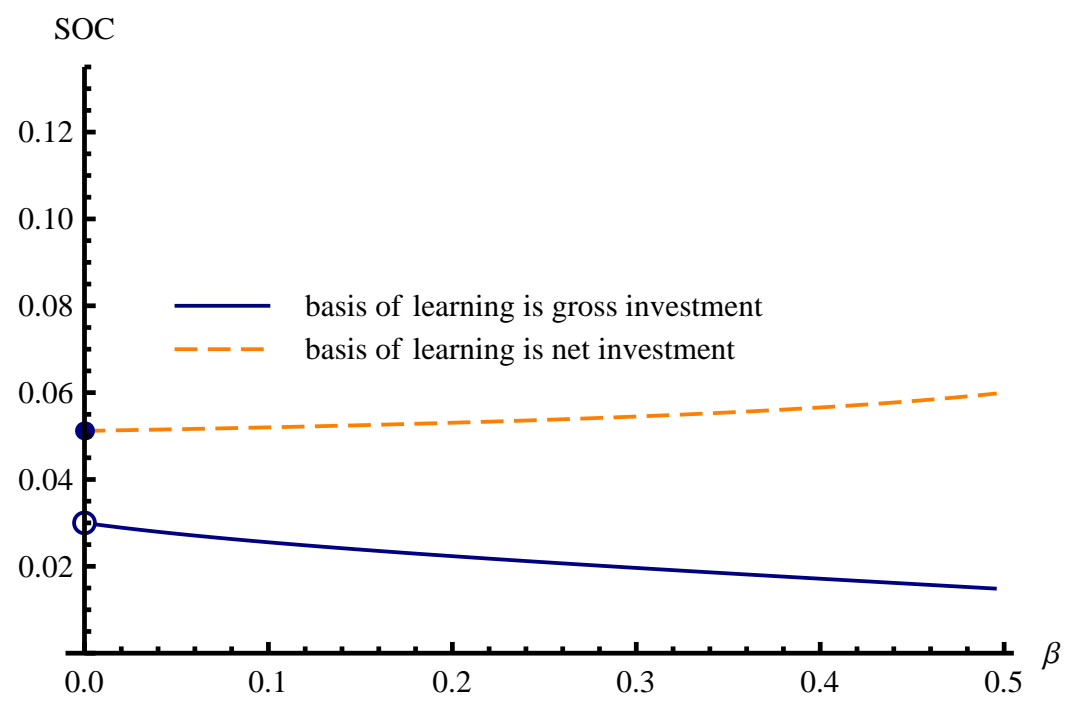

Figure 7: Speed of convergence as the disembodied learning parameter, $\beta$, rises and $\gamma$ is adjusted so as to maintain $g_{c}^{*}=0.02$, while $\alpha$ is adjusted so as to maintain $\alpha+(1-\alpha) \beta=0.5$. Note: $\lambda=0, \psi=0$.

ulation growth rate falls, respectively. ${ }^{21}$ As expected, this also holds in the present framework. At the same time, as documented in Appendix G, the qualitative patterns displayed by the graphs above go through for alternative values of $\theta$ and $n$, respectively. These patterns are also generally robust with respect to variation in values of the other background parameters, as long as restrictions (A1) and (A2) are observed. ${ }^{22}$ Moreover, both qualitatively and quantitatively similar results are obtained when the household sector is instead described within a Blanchard-Yaari type of overlapping generations framework.

\section{Conclusion}

Based on a dynamic general equilibrium model we have studied how the composition of technical progress, along three dimensions, affects the asymptotic speed of convergence. The analysis shows that the speed of convergence depends strongly and negatively on endogenous learning and on gross investment being the basis of learning rather than net investment. A rising degree of embodiment of technical

\footnotetext{
${ }^{21}$ See, e.g., Barro and Sala-i-Martin (2004, p. 112) and Turnovsky (2002).

${ }^{22}$ Sensitivity analysis w.r.t. $\alpha, \delta, \rho$, and $n$ is available from the authors upon request.
} 
change in the wake of the computer revolution is not likely to offset these effects.

A theoretical accomplishment of the analysis is the novel result, linked to the distinction between decomposable and indecomposable dynamics, that as soon as learning from gross investment becomes part of the growth engine, the asymptotic speed of convergence displays a discrete fall.

Overall our results point to a speed of convergence on the small side of $2 \%$ per year and possibly tending to a lower level in the future due to the rising importance of investment-specific learning in the wake of the computer revolution as the empirical evidence suggests.

\section{$7 \quad$ Appendix}

\section{A. Steady state}

By $(10)$, the steady state value of the consumption-capital ratio is $x^{*}=z^{*}-g_{K}^{*}-\delta$.

By substituting (27) and (23) into this expression, we get

$$
\begin{aligned}
x^{*}= & \frac{[(1-\alpha) \gamma+\alpha \psi] \theta-\{\alpha[1-(1-\alpha) \beta]-(1-\alpha)(1-\beta)\} \psi+(1-\alpha) \gamma[(1-\alpha) \lambda-\alpha]}{\alpha[(1-\alpha)(1-\beta)-\alpha \lambda]} \\
& +\frac{\{[(1-\alpha) \beta+\alpha \lambda] \theta+(1-\alpha)[(1-\alpha) \lambda-\alpha]\} n}{\alpha[(1-\alpha)(1-\beta)-\alpha \lambda]}+\frac{\rho+(1-\alpha) \delta}{\alpha} .
\end{aligned}
$$

For the proof of (vi) of Proposition 1 we need:

Lemma A1. Assume (A1) and (A2). Then $g_{K}^{*}=(1+\lambda) g_{Y}^{*}+\psi$.

Proof. From (23) follows

$$
\begin{aligned}
g_{K}^{*}-\psi & =\frac{[1-(1-\alpha) \beta] \psi+(1+\lambda)(1-\alpha)(\gamma+n)-[(1-\alpha)(1-\beta)-\alpha \lambda] \psi}{(1-\alpha)(1-\beta)-\alpha \lambda} \\
& =\frac{(1+\lambda) \alpha \psi+(1+\lambda)(1-\alpha)(\gamma+n)}{(1-\alpha)(1-\beta)-\alpha \lambda}=(1+\lambda) g_{Y}^{*},
\end{aligned}
$$

by $(22)$. 


\section{B. Eigenvalues}

Assume (A1) and (A2). Then, by Proposition $1, s^{*} x^{*} z^{*} u^{*}>0$. The Jacobian matrix associated with the system (21), (14), and (15) evaluated in the steady state, is $\mathbf{A}=$

$$
\left[\begin{array}{ccc}
x^{*}\left(1-\frac{\theta-1}{\theta} \lambda \frac{u^{*}}{z^{*}}\right) & x^{*}\left(\frac{\alpha}{\theta}-1+\frac{\theta-1}{\theta} \lambda \frac{x^{*} u^{*}}{z^{* 2}}\right) & x^{*} \frac{\theta-1}{\theta} \lambda s^{*} \\
z^{*}\left[1-\alpha-((1-\alpha) \beta+\lambda) \frac{u^{*}}{z^{*}}\right] & z^{*}\left[\alpha-1+((1-\alpha) \beta+\lambda) \frac{x^{*} u^{*}}{z^{* 2}}\right] & z^{*}((1-\alpha) \beta+\lambda) s^{*} \\
u^{*}\left[-\alpha+(1-(1-\alpha) \beta) \frac{u^{*}}{z^{*}}\right] & u^{*}\left[\alpha-(1-(1-\alpha) \beta) \frac{x^{*} u^{*}}{z^{* 2}}\right] & -u^{*}(1-(1-\alpha) \beta) s^{*}
\end{array}\right],
$$

where $s^{*} \equiv 1-x^{*} / z^{*}$. The expression for the determinant can be reduced to

$$
\operatorname{det} \mathbf{A}=\frac{\alpha}{\theta}[(1-\alpha)(1-\beta)-\alpha \lambda] s^{*} x^{*} z^{*} u^{*}>0,
$$

where the inequality follows from the parameter restriction in (6) and the positivity of $s^{*} x^{*} z^{*} u^{*}$. Thus either there are two eigenvalues with negative real part and one positive eigenvalue or all three eigenvalues, $\eta_{1}, \eta_{2}$, and $\eta_{3}$, have positive real part. Since the dynamic system has two pre-determined variables, $z$ and $u$, and one jump variable, $x$, saddle-point stability requires that the latter possibility can be ruled out. And indeed it can. Consider

$$
b \equiv \sum_{j>i}\left|\begin{array}{ll}
a_{i i} & a_{i j} \\
a_{j i} & a_{j j}
\end{array}\right|
$$

where $a_{i j}$ is the element in the $i$ 'th row and $j$ 'th column of A. From matrix algebra we know that $b=\eta_{1} \eta_{2}+\eta_{1} \eta_{3}+\eta_{2} \eta_{3}$. By Lemma B1 below, $b<0$, and so the possibility that all three eigenvalues have positive real part can be ruled out. ${ }^{23}$

Lemma B1. Assume (A1) and (A2). Then $b<0$.

Proof. From the definition of $\mathbf{A}$ follows

$$
\begin{aligned}
\left|\begin{array}{ll}
a_{11} & a_{12} \\
a_{21} & a_{22}
\end{array}\right|= & \left\{-\frac{\alpha}{\theta}(1-\alpha)-\left[(1-\alpha) \beta+\frac{\lambda}{\theta}\right] s^{*} \frac{u^{*}}{z^{*}}+[(1-\alpha) \beta+\lambda] \frac{\alpha}{\theta} \frac{u^{*}}{z^{*}}\right. \\
& \left.+\left(\frac{1}{\theta}-1\right) \alpha \lambda s^{*} \frac{u^{*}}{z^{*}}\right\} x^{*} z^{*}, \\
\left|\begin{array}{ll}
a_{11} & a_{13} \\
a_{31} & a_{33}
\end{array}\right|= & {\left[(1-\alpha) \beta-1+\left(\frac{1}{\theta}-1\right) \alpha\right] s^{*} x^{*} u^{*}, } \\
\left|\begin{array}{ll}
a_{22} & a_{23} \\
a_{32} & a_{33}
\end{array}\right|= & {[(1-\alpha)(1-\beta)-\alpha \lambda] s^{*} z^{*} u^{*} . }
\end{aligned}
$$

\footnotetext{
${ }^{23}$ Lemma B1 is a slight generalization of a similar result in Groth (2010).
} 
By summation and ordering,

$$
\begin{aligned}
b= & \left\{-\frac{\alpha}{\theta}(1-\alpha) x^{*}+\frac{1}{\theta}\left[\lambda\left(\alpha-s^{*}\right)+\alpha(1-\alpha) \beta\right] \frac{u^{*}}{z^{*}} x^{*}\right. \\
& \left.-s^{*} \frac{u^{*}}{z^{*}} x^{*}+[(1-\alpha)(1-\beta)-\alpha \lambda] s^{*} u^{*}\right\} z^{*} \\
= & \left\{\frac{1}{\theta}\left[-\alpha(1-\alpha)+\left(\lambda\left(\alpha-s^{*}\right)+\alpha(1-\alpha) \beta\right) \frac{u^{*}}{z^{*}}\right] x^{*}\right. \\
& \left.-\left[\frac{x^{*}}{z^{*}}-(1-\alpha)(1-\beta)+\alpha \lambda\right] s^{*} u^{*}\right\} z^{*} \\
< & \left\{\frac{1}{\theta(1+\lambda)}\left[-\alpha(1-\alpha)(1+\lambda)+\lambda\left(\alpha-s^{*}\right)+\alpha(1-\alpha) \beta\right] x^{*}\right. \\
& \left.-\left[\frac{x^{*}}{z^{*}}-(1-\alpha)(1-\beta)+\alpha \lambda\right] s^{*} u^{*}\right\} z^{*} \\
< & \left\{-\frac{1}{\theta(1+\lambda)}\left[\alpha((1-\alpha)(1-\beta)-\alpha \lambda)+\lambda s^{*}\right] x^{*}\right. \\
& \left.-[1-\alpha-(1-\alpha)(1-\beta)+\alpha \lambda] s^{*} u^{*}\right\} z^{*} \\
= & \left\{-\frac{1}{\theta(1+\lambda)}\left[\alpha((1-\alpha)(1-\beta)-\alpha \lambda)+\lambda s^{*}\right] x^{*}-[(1-\alpha) \beta+\alpha \lambda] s^{*} u^{*}\right\} z^{*}<0,
\end{aligned}
$$

where the first inequality is due to $s^{*}<\alpha$ and $(1+\lambda) u^{*} / z^{*}<1$ by (iv) and (vi) of Proposition 1 , respectively, the second inequality to $x^{*} / z^{*}=1-s^{*}>1-\alpha$, by (iv) of Proposition 1, and the last inequality to the restriction on $\lambda$ in (6).

\section{Local existence and uniqueness of a convergent solution}

From Appendix B follows that the steady state has a two-dimensional stable manifold. Our numerical simulations suggest that the cases of repeated real eigenvalues or complex conjugate eigenvalues never arise for parameter values within a reasonable range. Hence we concentrate on the case of two distinct real negative eigenvalues, $\eta_{1}$ and $\eta_{2}$, where $\eta_{1}<\eta_{2}<0$. Then any convergent solution is, in a neighborhood of $\left(x^{*}, z^{*}, u^{*}\right)$, approximately of the form given in (29) which we repeat here for convenience:

$$
x_{i t}=C_{1 i} e^{\eta_{1} t}+C_{2 i} e^{\eta_{2} t}+x_{i}^{*}, \quad i=1,2,3,
$$

where the constants $C_{1 i}$ and $C_{2 i}$ depend on initial conditions. Let $\mathbf{v}^{\mathbf{1}}=\left(v_{1}^{1}, v_{2}^{1}, v_{3}^{1}\right)$ 
be an eigenvector associated with $\eta_{1}$. That is, $\mathbf{v}^{\mathbf{1}} \neq(0,0,0)$ satisfies

$$
\begin{aligned}
& \left(a_{11}-\eta_{1}\right) v_{1}^{1}+a_{12} v_{2}^{1}+a_{13} v_{3}^{1}=0 \\
& a_{21} v_{1}^{1}+\left(a_{22}-\eta_{1}\right) v_{2}^{1}+a_{23} v_{3}^{1}=0 \\
& a_{31} v_{1}^{1}+a_{32} v_{2}^{1}+\left(a_{33}-\eta_{1}\right) v_{3}^{1}=0
\end{aligned}
$$

where one of the equations is redundant. Similarly, let $\mathbf{v}^{\mathbf{2}}=\left(v_{1}^{2}, v_{2}^{2}, v_{3}^{2}\right)$ be an eigenvector associated with $\eta_{2}$. Then, with $\eta_{1}$ replaced by $\eta_{2}$ in (34), these equations hold for $\left(v_{1}^{1}, v_{2}^{1}, v_{3}^{1}\right)$ replaced by $\left(v_{1}^{2}, v_{2}^{2}, v_{3}^{2}\right)$. Moreover, as $\eta_{1} \neq \eta_{2}, \mathbf{v}^{\mathbf{1}}$ and $\mathbf{v}^{\mathbf{2}}$ are linearly independent. The $C_{i}$ 's in (33) are related to this in the following way:

$$
C_{j i}=c_{j} v_{i}^{j}, \quad j=1,2, \quad i=1,2,3,
$$

where $c_{j}, j=1,2$, are constants to be determined by the given initial condition $\left(x_{20}, x_{30}\right)=\left(\bar{z}_{0}, \bar{u}_{0}\right)$.

Returning to our original variable notation $\left(x_{1 t}=x_{t}, x_{2 t}=z_{t}\right.$, and $\left.x_{3 t}=u_{t}\right)$, (33) together with (35) implies, for $t=0$ and $\left(z_{0}, u_{0}\right)=\left(\bar{z}_{0}, \bar{u}_{0}\right)$,

$$
\begin{aligned}
v_{1}^{1} c_{1}+v_{1}^{2} c_{2}-x_{0} & =-x^{*}, \\
v_{2}^{1} c_{1}+v_{2}^{2} c_{2}+0 & =\bar{z}_{0}-z^{*} \\
v_{3}^{1} c_{1}+v_{3}^{2} c_{2}+0 & =\bar{u}_{0}-u^{*}
\end{aligned}
$$

where $\bar{z}_{0}$ and $\bar{u}_{0}$ are given whereas $c_{1}, c_{2}$, and $x_{0}$ are the unknowns. For the steady state to be saddle-point stable the structure of $\mathbf{A}$ must be such that this system has a unique solution $\left(c_{1}, c_{2}, x_{0}\right)$. This is the case if and only if the vector $\mathbf{h}=$ $(-1,0,0)$ does not belong to the linear subspace, $S p\left(\mathbf{v}^{\mathbf{1}}, \mathbf{v}^{\mathbf{2}}\right)$, spanned by the linearly independent eigenvectors $\mathbf{v}^{\mathbf{1}}$ and $\mathbf{v}^{\mathbf{2}}$. Our claim is that this condition is satisfied. We prove this by showing that the opposite leads to a contradiction.

Suppose that, contrary to our claim, there exist constants $\alpha_{1}$ and $\alpha_{2}$ such that

$$
\alpha_{1} \mathbf{v}^{\mathbf{1}}+\alpha_{2} \mathbf{v}^{\mathbf{2}}=\mathbf{h}=\left(\begin{array}{c}
-1 \\
0 \\
0
\end{array}\right)
$$


Multiplying from the left by $\mathbf{A}$ gives

$$
\alpha_{1} \mathbf{A} \mathbf{v}^{\mathbf{1}}+\alpha_{2} \mathbf{A} \mathbf{v}^{\mathbf{2}}=\alpha_{1} \eta_{1} \mathbf{v}^{\mathbf{1}}+\alpha_{2} \eta_{2} \mathbf{v}^{\mathbf{2}}=\mathbf{A} \mathbf{h}=\left(\begin{array}{c}
-a_{11} \\
-a_{21} \\
-a_{31}
\end{array}\right)
$$

where we have used the definition of eigenvalues. By (37) follow $\alpha_{2} v_{2}^{2}=-\alpha_{1} v_{2}^{1}$ and $\alpha_{2} v_{3}^{2}=-\alpha_{1} v_{3}^{1}$. Substituting into (38) yields

$$
\begin{aligned}
& \alpha_{1} v_{2}^{1} \eta_{1}-\alpha_{1} v_{2}^{1} \eta_{2}=-a_{21} \\
& \alpha_{1} v_{3}^{1} \eta_{1}-\alpha_{1} v_{3}^{1} \eta_{2}=-a_{31}
\end{aligned}
$$

so that

$$
\begin{aligned}
& \alpha_{1} v_{2}^{1}=-\alpha_{2} v_{2}^{2}=\frac{a_{21}}{\eta_{2}-\eta_{1}} \\
& \alpha_{1} v_{3}^{1}=-\alpha_{2} v_{3}^{2}=\frac{a_{31}}{\eta_{2}-\eta_{1}}
\end{aligned}
$$

where $\eta_{2}-\eta_{1}>0$

Lemma C1. Assume (A1) and (A2). Then $a_{11}>0, a_{21}>0, a_{22}<0, a_{33}<0$, and $a_{31}+a_{32}>0$.

Proof. Assume (A1) and (A2). Then, by Proposition $1, s^{*} x^{*} z^{*} u^{*}>0$. From the definition of $\mathbf{A}$ in Appendix $\mathrm{B}$ we have, first, $a_{11}=x^{*}\left[1-\left(1-\theta^{-1}\right) \lambda u^{*} / z^{*}\right]>$ $x^{*}\left(1-\lambda u^{*} / z^{*}\right)>0$, where the last inequality follows from $u^{*} / z^{*}<1 /(1+\lambda)$, cf. (v) of Proposition 1; second, $a_{21}=z^{*}\left[1-\alpha-((1-\alpha) \beta+\lambda) u^{*} / z^{*}\right]>0$ by (v) of Proposition 1 and the restriction on $\lambda$ in (6); third, $a_{22}=z^{*}\left[\alpha-1+((1-\alpha) \beta+\lambda)\left(1-s^{*}\right) u^{*} / z^{*}\right]$ $=-a_{21}-z^{*}((1-\alpha) \beta+\lambda) s^{*} u^{*} / z^{*}<0$, since $a_{21}>0$; fourth, we immediately have $a_{33}<0$; finally, $a_{31}+a_{32}=u^{*}[1-(1-\alpha) \beta] s^{*} u^{*} / z^{*}>0$.

By Lemma $\mathrm{C} 1, a_{21} \neq 0$ and so (40) together with (39) implies that

$$
v_{3}^{1}=a_{31} v_{2}^{1} / a_{21}
$$

and that $v_{2}^{1} \neq 0$ (and $v_{2}^{2} \neq 0$ ). Multiplying the second equation in (34) by $a_{31}$ and the third by $a_{21}$ and subtracting yields

$$
\left[a_{31}\left(a_{22}-\eta_{1}\right)-a_{21} a_{32}\right] v_{2}^{1}+\left[a_{31} a_{23}-a_{21}\left(a_{33}-\eta_{1}\right)\right] v_{3}^{1}=0
$$


Substituting (41) into this, $v_{2}^{1}$ cancels out. Ordering gives

$$
a_{32} a_{21}^{2}-a_{23} a_{31}^{2}-a_{21} a_{31}\left(a_{22}-a_{33}\right)=0 .
$$

It remains to show that (42) implies a contradiction.

Let $k_{1} \equiv 1-(1-\alpha) \beta>0$ and $k_{2} \equiv(1-\alpha) \beta+\lambda \geq 0$. Insert the elements of $\mathbf{A}$ into the left-hand side of (42) to get $a_{32} a_{21}^{2}-a_{23} a_{31}^{2}-a_{21} a_{31}\left(a_{22}-a_{33}\right)$

$$
\begin{aligned}
& =z^{*} u^{*}\left\{\left(\alpha-k_{1} \frac{x^{*} u^{*}}{z^{* 2}}\right) z^{*}\left(1-\alpha-k_{2} \frac{u^{*}}{z^{*}}\right)^{2}\right. \\
& \left.-k_{2} s^{*} u^{*}\left(k_{1} \frac{u^{*}}{z^{*}}-\alpha\right)^{2}-\left(1-\alpha-k_{2} \frac{u^{*}}{z^{*}}\right)\left(k_{1} \frac{u^{*}}{z^{*}}-\alpha\right)\left[(\alpha-1) z^{*}+k_{1} s^{*} u^{*}+k_{2} \frac{x^{*} u^{*}}{z^{*}}\right]\right\} \\
& =s^{*} z^{*} u^{* 2} k_{1}\left\{(1-\alpha)\left[1-(1+\lambda) \frac{u^{*}}{z^{*}}\right]+\alpha k_{2} \frac{u^{*}}{z^{*}}\right\}>s^{*} z^{*} u^{* 2} k_{1} \alpha k_{2} \frac{u^{*}}{z^{*}} \geq 0,
\end{aligned}
$$

where the first inequality is implied by $\alpha<1$ and (v) of Proposition 1. Having hereby falsified (42), we conclude that $\mathbf{h} \notin S p\left(\mathbf{v}^{\mathbf{1}}, \mathbf{v}^{\mathbf{2}}\right)$, implying existence of a unique convergent solution.

\section{When A is indecomposable, generically the same asymptotic speed of convergence applies to all three variables in the dynamic system}

Consider an $n \times n$ matrix $\mathbf{M}, n \geq 2$. Let the element in the $i$ 'th row and $j$ 'th column of $\mathbf{M}$ be denoted $a_{i j}$. Let $S$ be a subset of the row (and column) indices $N=\{1,2, \ldots, n\}$ and let $S^{c}$ be the complement of $S$. Then $\mathbf{M}$ is defined as decomposable if there exists a subset $S$ of $N$ such that $a_{i j}=0$ for $i \in S, j \in S^{c}$. Thus, when the matrix $\mathbf{M}$ is decomposable, then by interchanging some rows as well as the corresponding columns it is possible to obtain a lower block-triangular matrix, that is, a matrix with a null submatrix in the upper right corner. A special case of a decomposable matrix $\mathbf{M}$ is the case where by interchanging some rows as well as the corresponding columns it is possible to obtain a lower triangular matrix, that is, a matrix with zeros everywhere above the main diagonal.

If $\mathbf{M}$ is decomposable, any subset $S$ of the row indices such that $a_{i j}=0$ for $i \in S$, $j \in S^{c}$, is called an independent subset. If a quadratic matrix is not decomposable, it is called indecomposable. 
By inspection of the Jacobian matrix A defined in Appendix B we check under what circumstances $\mathbf{A}$ is decomposable. We have $N=\{1,2,3\}$. Using Lemma C1 we first see that the only row number that can by itself be an independent subset is $\{1\}$, which requires $a_{12}=a_{13}=0$. This will hold if and only if $\lambda=0$ and $\theta=\alpha$. Next we check when a pair of rows constitutes an independent subset. If $\{1,2\}$ is an independent subset, we must have $a_{13}=a_{23}=0$. This will hold if and only if $\lambda=\beta=0$. The pair $\{2,3\}$ can not be an independent subset since $a_{21} \neq 0$, by Lemma C1. Finally, if $\{1,3\}$ should be an independent subset, we should have $a_{12}$ $=a_{32}=0$. It is easily shown that necessary (but not sufficient) for $a_{12}=0$ is that $\theta \leq \alpha$. And $a_{32}=0$ is only possible for very special combinations of parameter values involving all parameters of the system. So from a generic point of view we can rule out this case, which is not of much interest anyway because $\theta \leq \alpha$ is not empirically plausible.

We are left with two decomposable cases: Case $\mathfrak{D} 1: \lambda=0=\beta, \theta \neq \alpha$; and Case $\mathfrak{D} 2: \lambda=0, \beta \geq 0, \theta=\alpha$. These cases are treated in Appendix E.

Here we consider the complement of the union of these cases, that is, the case where $\lambda>0$ or $(\beta>0$ and $\theta \neq \alpha)$, implying that the Jacobian matrix $\mathbf{A}$ is generically indecomposable.

Regarding the eigenvalues of $\mathbf{A}$, as above we concentrate on the case of two distinct real negative eigenvalues, $\eta_{1}$ and $\eta_{2}$, where $\eta_{1}<\eta_{2}<0$, and one positive eigenvalue, $\eta_{3}$.

Lemma D1. Assume (A1) and (A2). Let $\mathbf{v}^{2}=\left(v_{1}^{2}, v_{2}^{2}, v_{3}^{2}\right)$ be an eigenvector associated with $\eta_{2}$, where $\eta_{1}<\eta_{2}<0$. If $\lambda>0$ or $(\beta>0$ and $\theta \neq \alpha)$, then $v_{2}^{2} \neq 0$, and, generically, $v_{i}^{2} \neq 0$, for $i=1,3$.

Proof. Assume (A1) and (A2) and that $\lambda>0$ or $(\beta>0$ and $\theta \neq \alpha)$. It immediately 
follows that $a_{23}>0$. By definition of $\eta_{2}$ and $\mathbf{v}^{2}$,

$$
\begin{aligned}
& \left(a_{11}-\eta_{2}\right) v_{1}^{2}+a_{12} v_{2}^{2}+a_{13} v_{3}^{2}=0 \\
& a_{21} v_{1}^{2}+\left(a_{22}-\eta_{2}\right) v_{2}^{2}+a_{23} v_{3}^{2}=0, \\
& a_{31} v_{1}^{2}+a_{32} v_{2}^{2}+\left(a_{33}-\eta_{2}\right) v_{3}^{2}=0 .
\end{aligned}
$$

That $v_{2}^{2} \neq 0$ is shown by contradiction. Suppose $v_{2}^{2}=0$. Then, by (43) and (44),

$$
\left[\begin{array}{cc}
a_{11}-\eta_{2} & a_{13} \\
a_{21} & a_{23}
\end{array}\right]\left(\begin{array}{l}
v_{1}^{2} \\
v_{3}^{2}
\end{array}\right)=\left(\begin{array}{l}
0 \\
0
\end{array}\right),
$$

where $v_{1}^{2} \neq 0$ or $v_{3}^{2} \neq 0$, since $\mathbf{v}^{2}$ is an eigenvector. Consequently, the determinant of the $2 \times 2$ matrix must be vanishing, i.e., $\left(a_{11}-\eta_{2}\right) a_{23}-a_{21} a_{13}=0$. But, considering matrix A we have, after ordering,

$$
\left(a_{11}-\eta_{2}\right) a_{23}-a_{21} a_{13}=\frac{s^{*} z^{*}}{\theta}\left\{(1-\alpha) \beta \theta\left(x^{*}-\eta_{2}\right)+\lambda\left[(1-\alpha+\alpha \theta) x^{*}-\theta \eta_{2}\right]\right\}>0
$$

where the inequality follows from $\eta_{2}<0$ and the assumption that $\lambda>0$ or $\beta>0$. From this contradiction we conclude that $v_{2}^{2} \neq 0$.

Now suppose $v_{1}^{2}=0$. Then, by (43) and (44),

$$
\left[\begin{array}{cc}
a_{12} & a_{13} \\
a_{22}-\eta_{2} & a_{23}
\end{array}\right]\left(\begin{array}{l}
v_{2}^{2} \\
v_{3}^{2}
\end{array}\right)=\left(\begin{array}{l}
0 \\
0
\end{array}\right) .
$$

Since $v_{2}^{2} \neq 0$, the determinant of the $2 \times 2$ matrix must be vanishing:

$$
a_{12} a_{23}-a_{13}\left(a_{22}-\eta_{2}\right)=0
$$

But, as noted above, $a_{23}>0$; and since by assumption, if $\lambda=0$, we have $\theta \neq \alpha, a_{12}$ and $a_{13}$ cannot be nil at the same time. Consequently, in no dense open subset in the relevant parameter space does (46) hold. This proves the genericity of $v_{1}^{2} \neq 0$.

Finally, suppose $v_{3}^{2}=0$. Then, by (43) and (45),

$$
\left[\begin{array}{cc}
a_{11}-\eta_{2} & a_{12} \\
a_{31} & a_{32}
\end{array}\right]\left(\begin{array}{l}
v_{1}^{2} \\
v_{2}^{2}
\end{array}\right)=\left(\begin{array}{l}
0 \\
0
\end{array}\right) .
$$

Since $v_{2}^{2} \neq 0$, the determinant of the $2 \times 2$ matrix must be vanishing:

$$
\left(a_{11}-\eta_{2}\right) a_{32}-a_{31} a_{12}=0 .
$$


But $a_{11}-\eta_{2}>0$ and, by Lemma C1, $a_{31}$ and $a_{32}$ cannot be nil at the same time. Consequently, in no dense open subset in the relevant parameter space does (47) hold. This proves the genericity of $v_{3}^{2} \neq 0$.

Lemma D2. Assume (A1) and (A2). Let $x_{i 0} \neq x_{i}^{*}, i=1,2,3$. If $\lambda>0$ or $(\beta>0$ and $\theta \neq \alpha$ ), then $c_{2}$ in (35) differs generically from 0 .

Proof. In Appendix $\mathrm{C}$ we showed that (36) has a unique solution $\left(c_{1}, c_{2}, x_{0}\right)$. By Cramer's rule

$$
c_{2}=-\frac{\left(z_{0}-z^{*}\right) v_{3}^{1}-\left(u_{0}-u^{*}\right) v_{2}^{1}}{v_{2}^{1} v_{3}^{2}-v_{2}^{2} v_{3}^{1}},
$$

where $v_{2}^{1} v_{3}^{2}-v_{2}^{2} v_{3}^{1} \neq 0$, that is, $\left(v_{2}^{1}, v_{3}^{1}\right) \neq(0,0)$ and $\left(v_{2}^{2}, v_{3}^{2}\right) \neq(0,0)$. Let $z_{0} \neq z^{*}$ and $u_{0} \neq u^{*}$. Suppose $c_{2}=0$. Then $\left(z_{0}-z^{*}\right) v_{3}^{1}=\left(u_{0}-u^{*}\right) v_{2}^{1}$, which is possible only if $v_{2}^{1} \neq 0, v_{3}^{1} \neq 0$, and the pair $\left(z_{0}, u_{0}\right)$ satisfies $\left(z_{0}-z^{*}\right) /\left(u_{0}-u^{*}\right)=v_{2}^{1} / v_{3}^{1}$. Such pairs, however, do not constitute a dense open subset in the $(z, u)$-plane, as was to be shown.

Combining Lemma D1 and D2 we have that when (A1) and (A2) hold together with $\lambda>0$ or $(\beta>0$ and $\theta \neq \alpha)$, then generically $C_{2 i}=c_{2} v_{i}^{2} \neq 0, i=1,2,3$. In the light of (30) it follows that in this case the same asymptotic speed of convergence, $-\eta_{2}$, applies to all three variables in the dynamic system. This proves Proposition 3.

\section{E. Discontinuity of the dominant eigenvalue for the $\mathrm{x}$ and $\mathrm{z}$ dynamics when learning disappears}

We assume throughout that (A1) and (A2) hold so that, by Proposition 1, $x^{*}$, $z^{*}, u^{*}$, and $s^{*}$ are all strictly positive.

Decomposable case $\mathfrak{D} 1: \lambda=0=\beta, \theta \neq \alpha$. In this case $a_{13}=0=a_{23}$. So the Jacobian matrix $\mathbf{A}$ is lower block-triangular, implying that its eigenvalues coincide with the eigenvalues of the upper left $2 \times 2$ submatrix on the main diagonal of $\mathbf{A}$ and the lower right diagonal element, $a_{33}<0$. Let $\mathbf{A}_{11}$ denote the upper left $2 \times 2$ submatrix. 
Decomposable case $\mathfrak{D} 2: \lambda=0, \beta \geq 0, \theta=\alpha$. In this case (and only in this case) $a_{12}=0=a_{13}$. So $\mathbf{A}$ is again lower block-triangular, but this time with the positive eigenvalue equal to $a_{11}=x^{*}>0$, whereas the two negative eigenvalues are associated with the lower right $2 \times 2$ submatrix of $\mathbf{A}$. Let this submatrix be denoted $\mathbf{A}_{\mathbf{2 2}}$. As long as $\beta>0, a_{23} \neq 0$ and $\mathbf{A}$ is not further decomposable. In case $\beta=0$, also $a_{23}=0$. Then $\mathbf{A}_{\mathbf{2 2}}$, hence also $\mathbf{A}$, is lower triangular with the eigenvalues appearing on the main diagonal.

As a preparation for the proof of Proposition 4, which involves both case $\mathfrak{D} 1$ and $\mathfrak{D} 2$, we need three lemmas concerning case $\mathfrak{D} 1$. For case $\mathfrak{D} 1$ we have

$$
\mathbf{A}=\left[\begin{array}{cccc} 
& & & 0 \\
& \mathbf{A}_{11} & & 0 \\
a_{31} & & a_{32} & a_{33}
\end{array}\right]=\left[\begin{array}{ccc}
x^{*} & \left(\frac{\alpha}{\theta}-1\right) x^{*} & 0 \\
(1-\alpha) z^{*} & (\alpha-1) z^{*} & 0 \\
\left(\frac{u^{*}}{z^{*}}-\alpha\right) u^{*} & \left(\alpha-\frac{x^{*} u^{*}}{z^{* 2}}\right) u^{*} & -s^{*} u^{*}
\end{array}\right]
$$

The submatrix $\mathbf{A}_{\mathbf{1 1}}$ has determinant $\operatorname{det} \mathbf{A}_{\mathbf{1 1}}=-(1-\alpha) \frac{\alpha}{\theta} x^{*} z^{*}<0$. The eigenvalues are $\bar{\eta}_{1}$ and $\bar{\eta}_{3}$, where $\bar{\eta}_{1}<0<\bar{\eta}_{3}$. The third eigenvalue of $\mathbf{A}$ is $\bar{\eta}_{2}=-s^{*} u^{*}=-g_{Y}^{*}<$ 0 . For realistic parameter values we have $\bar{\eta}_{1}<\bar{\eta}_{2}<0$.

Lemma E1. Let $\lambda=0=\beta$ and $\theta \neq \alpha$. Let $z_{0}=\bar{z}_{0}>0$ be given. Then the unique convergent approximating solution for the $(x, z)$ subsystem is

$$
\begin{aligned}
& x_{t}=c v_{1}^{1} e^{\bar{\eta}_{1} t}+x^{*}, \\
& z_{t}=c v_{2}^{1} e^{\bar{\eta}_{1} t}+z^{*},
\end{aligned}
$$

where $\bar{\eta}_{1}$ is the negative eigenvalue of $\mathbf{A}_{\mathbf{1 1}}, v_{1}^{1}=1, v_{2}^{1}=-\left(x^{*}-\bar{\eta}_{1}\right) / a_{12} \neq 0$, and $c=\left(\bar{z}_{0}-z^{*}\right) / v_{2}^{1}$.

Proof. From Lemma C1 we know that $a_{21} \neq 0$ and since $\lambda=0$ is combined with $\theta \neq \alpha, a_{12} \neq 0$. So $\mathbf{A}_{11}$ is not decomposable. As $x^{*}>0$ and $\bar{\eta}_{1}<0$, we have $a_{12} v_{2}^{1}=-\left(x^{*}-\bar{\eta}_{1}\right)<0$, which implies $v_{2}^{1} \neq 0$. So $c=\left(\bar{z}_{0}-z^{*}\right) / v_{2}^{1}$ is well-defined and ensures, when combined with (50), that $z_{0}=\bar{z}_{0}$. Finally, since $x^{*}=a_{11}$, by construction $\left(v_{1}^{1}, v_{2}^{1}\right)$ satisfies the equation $\left(a_{11}-\bar{\eta}_{1}\right) v_{1}^{1}+a_{12} v_{2}^{1}=0$. Thus, $\left(v_{1}^{1}, v_{2}^{1}\right)$ $\neq(0,0)$ is an eigenvector of $A_{11}$ associated with $\bar{\eta}_{1}$; and (49)-(50) thereby constitutes the unique convergent approximating solution for the $(x, z)$ subsystem. 
Lemma E2. Let $\lambda=0=\beta$ and $\theta \neq \alpha$. Let the two negative eigenvalues of A, $\bar{\eta}_{1}$ and $\bar{\eta}_{2}$, satisfy $\bar{\eta}_{1}<\bar{\eta}_{2}<0$. Define $\mathbf{v}^{\mathbf{1}}=\left(v_{1}^{1}, v_{2}^{1}, v_{3}^{1}\right)$, where $\left(v_{1}^{1}, v_{2}^{1}\right)$ is as given in Lemma E1, and $v_{3}^{1}=\left(a_{31} v_{1}^{1}+a_{32} v_{2}^{1}\right) /\left(\bar{\eta}_{1}-a_{33}\right)$. Then $\mathbf{v}^{\mathbf{1}}$ is an eigenvector of $\mathbf{A}$ associated with the eigenvalue $\bar{\eta}_{1}$. Further, $\mathbf{v}^{\mathbf{2}}=\left(v_{1}^{2}, v_{2}^{2}, v_{3}^{2}\right)=(0,0,1)$ is an eigenvector of $\mathbf{A}$ associated with the eigenvalue $\bar{\eta}_{2}$.

Proof. Since $a_{33}=\bar{\eta}_{2}>\bar{\eta}_{1}, \bar{\eta}_{1}-a_{33}<0$. Then $v_{3}^{1}$ is well-defined and by construction $\mathbf{v}^{\mathbf{1}}$ satisfies (34) with $\eta_{1}=\bar{\eta}_{1}$ in view of $a_{13}=a_{23}=0$. Let $\mathbf{w}=\left(w_{1}, w_{2}, w_{3}\right)$ be an arbitrary eigenvector of $\mathbf{A}$ associated with the eigenvalue $\bar{\eta}_{2}$ :

$$
\begin{aligned}
\left(a_{11}-\bar{\eta}_{2}\right) w_{1}+a_{12} w_{2}+0 & =0, \\
a_{21} w_{1}+\left(a_{22}-\bar{\eta}_{2}\right) w_{2}+0 & =0, \\
a_{31} w_{1}+a_{32} w_{2}+\left(a_{33}-\bar{\eta}_{2}\right) w_{3} & =0 .
\end{aligned}
$$

The eigenvalues of $\mathbf{A}_{11}$ are $\bar{\eta}_{1}<0$ and $\bar{\eta}_{3}>0$, and since $\bar{\eta}_{1}<\bar{\eta}_{2}<0, \bar{\eta}_{2}$ cannot be an eigenvalue of $\mathbf{A}_{11}$. Hence, $w_{1}=0=w_{2}$. As $\bar{\eta}_{2}=a_{33}$, this implies that $w_{3} \neq 0$ is arbitrary and can be set equal to 1 . Thereby $\mathbf{v}^{\mathbf{2}}=\mathbf{w}$.

Lemma E3. Let $\lambda=0=\beta$ and $\theta \neq \alpha$. Let $z_{0}=\bar{z}_{0}>0$ and $u_{0}=\bar{u}_{0}>0$ be given. Let $c$ be defined as in Lemma E1 and $\mathbf{v}^{\mathbf{1}}$ and $\mathbf{v}^{\mathbf{2}}$ as in Lemma E2. Then the unique convergent approximating solution for the total system is given by (49), (50), and

$$
u_{t}=c_{1} v_{3}^{1} e^{\bar{\eta}_{1} t}+c_{2} v_{3}^{2} e^{\bar{\eta}_{2} t}+u^{*}
$$

with $c_{1}=c=\left(\bar{z}_{0}-z^{*}\right) / v_{2}^{1}$ and $c_{2}=\bar{u}_{0}-u^{*}-c_{1} v_{3}^{1}$. The speed of convergence of $x$ and $z$ is $-\bar{\eta}_{1}$, whereas that of $u$ is $-\bar{\eta}_{2}$.

Proof. In Lemma E2 it was shown that $\mathbf{v}^{\mathbf{1}}$ and $\mathbf{v}^{\mathbf{2}}$ are eigenvectors of $\mathbf{A}$ associated with the eigenvalues $\bar{\eta}_{1}$ and $\bar{\eta}_{2}$, respectively. We show that the solution formula (33) with $\eta_{1}=\bar{\eta}_{1}, \eta_{2}=\bar{\eta}_{2}$, and $C_{j i}=c_{j} v_{i}^{j}, j=1,2, i=1,2,3$, for all $t \geq 0$ implies the proposed solution. In view of $c_{1}=c=\left(\bar{z}_{0}-z^{*}\right) / v_{2}^{1}$ and $v_{1}^{2}=0$, (33) for $i=1$ is the same as (49). In view of $c_{1}=c$ and $v_{2}^{2}=0,(33)$ for $i=2$ is the same as (50). It follows that $x$ and $z$ share the same speed of convergence, $-\bar{\eta}_{1}$. Finally, in view 
of $c_{2}=\bar{u}_{0}-u^{*}-c_{1} v_{3}^{1}$ and $v_{3}^{2}=1$, (33) for $i=3$ is the same as (51). It remains to show that $\bar{\eta}_{2}$ is the dominant eigenvalue for the dynamics of $u$. Since $\bar{\eta}_{1}<\bar{\eta}_{2}<0$, this is so if $C_{23} \equiv c_{2} v_{3}^{2} \neq 0$ generically. As $v_{3}^{2}=1$,

$$
c_{2} v_{3}^{2}=c_{2}=\bar{u}_{0}-u^{*}-c_{1} v_{3}^{1}=\bar{u}_{0}-u^{*}-\left(\bar{z}_{0}-z^{*}\right) v_{3}^{1} / v_{2}^{1},
$$

by the definition of $c_{1}$. Let $\bar{u}_{0} \neq u^{*}$ and $\bar{z}_{0} \neq z^{*}$. Suppose $c_{2}=0$. Then $\left(\bar{z}_{0}-\right.$ $\left.z^{*}\right) v_{3}^{1} / v_{2}^{1}=\bar{u}_{0}-u^{*}$. Pairs $\left(\bar{z}_{0}, \bar{u}_{0}\right)$ satisfying this do not, however, constitute a dense open subset in the $(z, u)$-plane. Hence $c_{2} v_{3}^{2}\left(=c_{2}\right) \neq 0$ generically, as was to be shown.

Proof of Proposition 4 of Section 3.4. It is given that when $\lambda=0=\beta$ and $\theta \neq \alpha$, the eigenvalues of $\mathbf{A}$ are real numbers, $\bar{\eta}_{1}, \bar{\eta}_{2}$, and $\bar{\eta}_{3}$, that satisfy $\bar{\eta}_{1}<\bar{\eta}_{2}$ $<0<\bar{\eta}_{3}$. Similarly, when $\lambda=0=\beta$ together with $\theta=\alpha$, the eigenvalues of $\mathbf{A}$ are real numbers, $\tilde{\eta}_{1}, \tilde{\eta}_{2}$, and $\tilde{\eta}_{3}$, that satisfy $\tilde{\eta}_{1}<\tilde{\eta}_{2}<0<\tilde{\eta}_{3}$.

(i): Suppose $\theta \neq \alpha$ and that $\lambda$ or $\beta$ (or both) are strictly positive but close to zero. By hyperbolicity of the steady state, the eigenvalues of $\mathbf{A}, \eta_{1}, \eta_{2}$, and $\eta_{3}$, are still real and, by continuity, close to $\bar{\eta}_{1}, \bar{\eta}_{2}$, and $\bar{\eta}_{3}$. Thus, maintaining numbering in accordance with size, we have $\eta_{1} \approx \bar{\eta}_{1}<\eta_{2} \approx \bar{\eta}_{2}<0<\eta_{3} \approx \bar{\eta}_{3}$. In view of $\theta \neq \alpha$, as long as $\lambda>0$ or $\beta>0$, Proposition 3 applies. So the same asymptotic speed of convergence, $-\eta_{2}$, applies to all three variables. Let $(\beta, \lambda) \rightarrow(0,0)^{+}$. Then $-\eta_{2} \rightarrow-\bar{\eta}_{2}$. In the limit Lemma E3 applies, that is, the equilibrium path for $x$ and $z$ is given by (49) and (50), respectively. Consequently, in the limit the speed of convergence of $x$ and $z$ shifts from the value $-\bar{\eta}_{2}$ to the value $-\bar{\eta}_{1}$.

(ii): Let $\theta=\alpha$ and $\beta=0$. As long as $\lambda>0, \mathbf{A}$ is indecomposable. Let $\lambda \rightarrow 0^{+}$. In the limit $\mathbf{A}$ takes the form given in (48) with $a_{12}=0$, that is, $\mathbf{A}$ becomes lower triangular with eigenvalues $\tilde{\eta}_{3}=x^{*}>0, \tilde{\eta}_{1}=(\alpha-1) z^{*}<0$, and $\tilde{\eta}_{2}=-g_{Y}^{*}<0$ where, by assumption, $\tilde{\eta}_{1}<\tilde{\eta}_{2}$. As long as $\lambda>0$, but close to zero, an argument analogue to that under (i) applies, except that in the limit it is only $z$ that shifts to a higher finite speed of convergence. The jump variable $x$ becomes in the limit independent of both $z$ and $u$. Thus $x$ becomes free to adjust instantaneously to its 
steady state value; that is, in the limit the speed of convergence of $x$ is infinite.

(iii): Let $\theta=\alpha$ and $\lambda=0$. Then, $a_{12}=a_{13}=0$. Even for $\beta>0$ the dynamic system belongs to the decomposable case $\mathfrak{D} 2$ described above, and the jump variable $x$ is independent of the dynamics of $z$ and $u$. So the speed of convergence of $x$ is infinite even for $\beta>0$ and remains so in the limit for $\beta \rightarrow 0^{+}$. But the $(z, u)$ dynamics is governed jointly by $\eta_{1} \approx \tilde{\eta}_{1}$ and $\eta_{2} \approx \tilde{\eta}_{2}$ as long as $\beta$ is strictly positive but close to zero, where $\tilde{\eta}_{1}<\tilde{\eta}_{2}<0$. In the limit for $\beta \rightarrow 0^{+}$, however, $\mathbf{A}$ becomes lower triangular and so the movement of $z$ ceases to be influenced by the slow adjustment of $u$ and is governed only by the eigenvalue $\tilde{\eta}_{1}=(\alpha-1) z^{*}$. The speed of convergence of $z$ thus jumps from $-\tilde{\eta}_{2}$ to the higher value $-\tilde{\eta}_{1}$.

\section{F. Saddle-point stability when learning is based on net investment}

When learning is based on net investment, the dynamic system becomes twodimensional, cf. the formulas for $g_{x}$ and $g_{z}$ in Section 4. To avoid explosive growth the parameter values are restricted as follows:

$$
0 \leq \lambda<(1-\alpha)(1-\beta)
$$

The Jacobian matrix evaluated in steady state is

$$
\mathbf{B}=\left[\begin{array}{cc}
x^{*}\left(1-\frac{\theta-1}{\theta} \lambda\right) & x^{*}\left(\frac{\alpha}{\theta}+\frac{\theta-1}{\theta} \lambda-1\right) \\
z^{*}[(1-\alpha)(1-\beta)-\lambda] & -z^{*}[(1-\alpha)(1-\beta)-\lambda]
\end{array}\right] .
$$

We find $\operatorname{det} \mathbf{B}=-\frac{\alpha}{\theta}[(1-\alpha)(1-\beta)-\lambda] x^{*} z^{*}<0$, where the inequality is implied by the parameter restriction $(*)$. Thus the eigenvalues, $\eta_{1}$ and $\eta_{2}$, differ in sign, and the steady state is saddle-point stable.

The non-trivial steady state, $\left(x^{*}, z^{*}\right)$, has consumption-capital ratio

$$
x^{*}=z^{*}-\delta-\frac{(1-\alpha)(\gamma+n)+\psi}{\alpha[(1-\alpha)(1-\beta)-\lambda]}
$$

and output-capital ratio

$$
\begin{aligned}
z^{*}= & \frac{\theta[(1-\alpha) \gamma+\alpha \psi]+(1-\alpha)[\lambda \gamma+(1-\beta) \psi+\theta(\beta \psi-\lambda \gamma)]}{\alpha[(1-\alpha)(1-\beta)-\lambda]} \\
& +\frac{\{\theta[(1-\alpha) \beta+\alpha \lambda]+(1-\alpha) \lambda\} n}{\alpha[(1-\alpha)(1-\beta)-\lambda]}+\frac{\delta+\rho}{\alpha} .
\end{aligned}
$$




\section{G. Simulations}

The numerical results in this appendix refer to the benchmark model with learning based on gross investment. "Speed of convergence" refers to the common speed of convergence of $x$ and $z$, i.e., $\sigma_{x}=\sigma_{z}$. By Proposition 4 , in the absence of learning, $\sigma_{u} \neq \sigma_{i}, i \in\{x, z\}$. In the tables, numbers in parentheses indicate the speed of convergence, in percentage points, of $u$ in the absence of learning. Unless otherwise specified, values of the background parameters are the baseline values specified in Table 2 of the text. The range of the parameter appearing in the first column of the tables is limited to values not requiring the adjusting variable to take on a negative value to maintain $g_{c}^{*}=0.02$. 
TABLE A

SPEed of CONVERGENCE AS THE EMBodied LEARNing PARAMETER, $\lambda$, RISES AND $\gamma$ IS ADJUSTED SO AS TO MAINTAIN $g_{c}^{*}=0.02$.

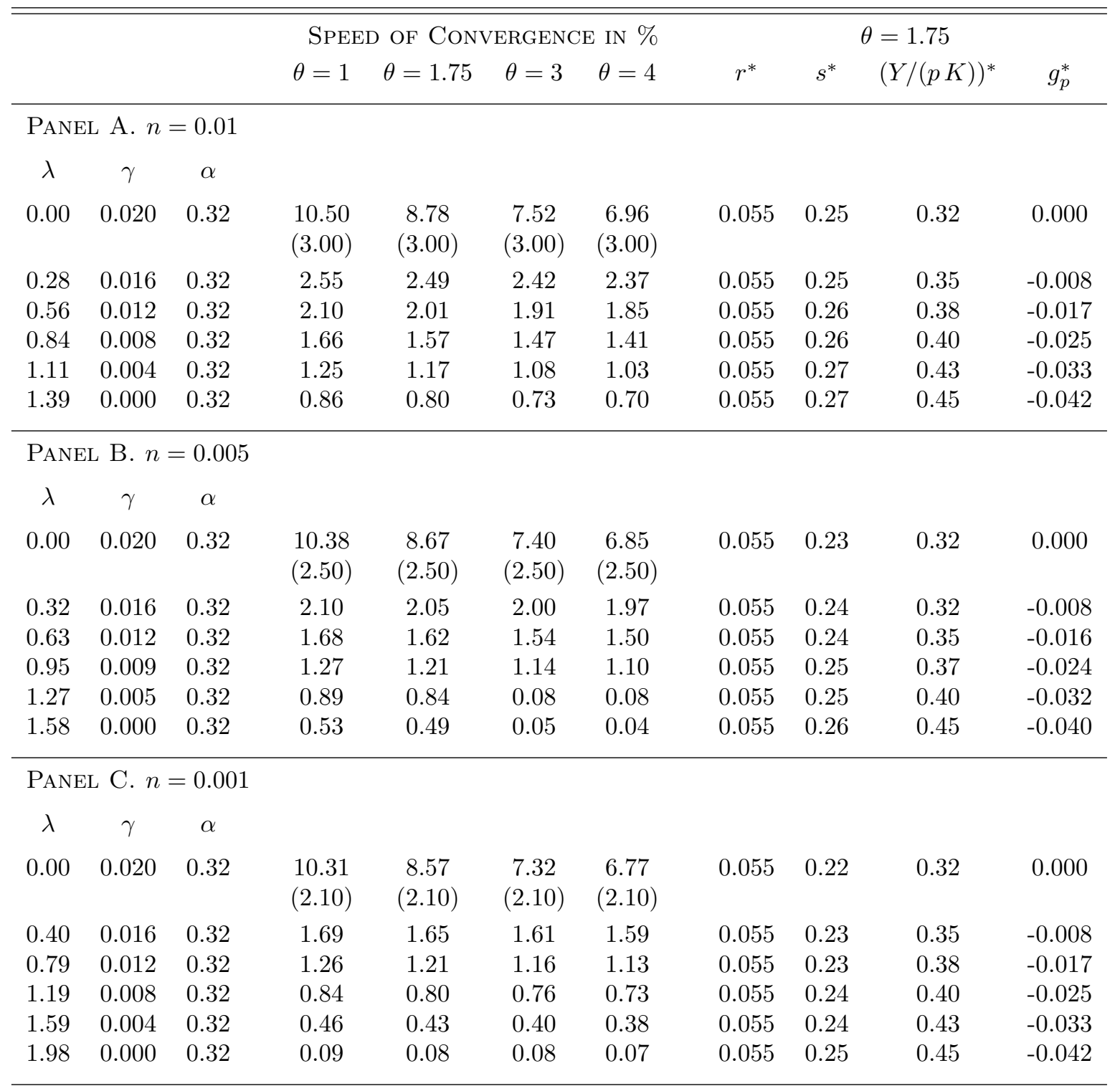

Note: $\beta=0, \psi=0$. When $\lambda=0, u$ converges with a lower speed than $(x, z)$. This lower speed is shown in brackets. 
TABLE B

Speed of CONVERGEnce AS the EMbodied Learning Parameter, $\lambda$, Rises AND $\psi$ IS ADJUSTED SO AS TO MAINTAIN $g_{c}^{*}=0.02$

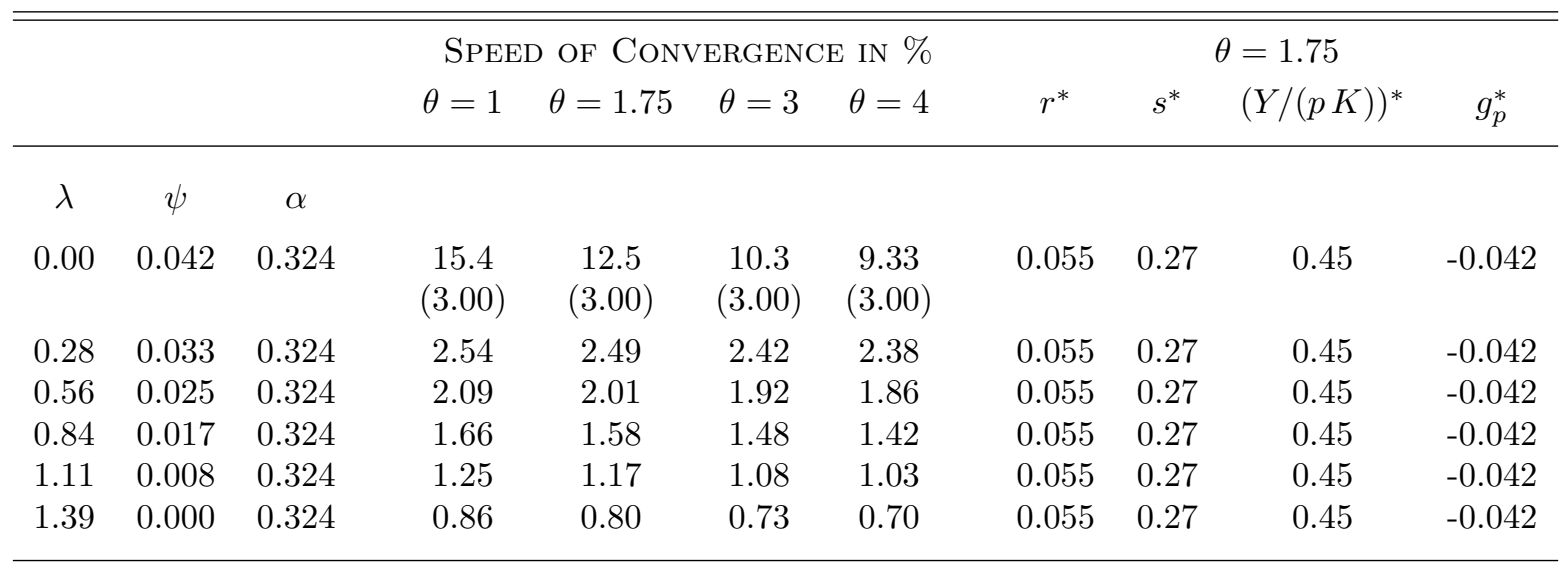

NotE: $\beta=0, \gamma=0$.

TABLE C

Speed of Convergence as the disembodied learning Parameter, $\beta$, RISES AND $\gamma$ IS ADJUSTED SO AS TO MAINTAIN $g_{c}^{*}=0.02$

\begin{tabular}{|c|c|c|c|c|c|c|c|c|c|c|}
\hline & & & \multicolumn{4}{|c|}{ Speed of Convergence in $\%$} & \multicolumn{4}{|c|}{$\theta=1.75$} \\
\hline & & & $\theta=1$ & $\theta=1.75$ & $\theta=3$ & $\theta=4$ & $r^{*}$ & $s^{*}$ & $(Y /(p K))^{*}$ & $g_{p}^{*}$ \\
\hline \multicolumn{11}{|c|}{ Panel A. } \\
\hline$\beta$ & $\gamma$ & $\alpha$ & & & & & & & & \\
\hline 0.00 & 0.020 & 0.32 & $\begin{array}{l}10.48 \\
(3.00)\end{array}$ & $\begin{array}{c}8.78 \\
(3.00)\end{array}$ & $\begin{array}{c}7.52 \\
(3.00)\end{array}$ & $\begin{array}{c}6.96 \\
(3.00)\end{array}$ & 0.055 & 0.25 & 0.32 & 0.0 \\
\hline 0.13 & 0.016 & 0.32 & 2.59 & 2.51 & 2.42 & 2.36 & 0.055 & 0.25 & 0.32 & 0.0 \\
\hline 0.27 & 0.012 & 0.32 & 2.17 & 2.05 & 1.90 & 1.82 & 0.055 & 0.25 & 0.32 & 0.0 \\
\hline 0.40 & 0.008 & 0.32 & 1.76 & 1.61 & 1.45 & 1.37 & 0.055 & 0.25 & 0.32 & 0.0 \\
\hline 0.53 & 0.004 & 0.32 & 1.35 & 1.20 & 1.10 & 0.99 & 0.055 & 0.25 & 0.32 & 0.0 \\
\hline 0.67 & 0.000 & 0.32 & 0.95 & 0.82 & 0.71 & 0.66 & 0.055 & 0.25 & 0.32 & 0.0 \\
\hline \multicolumn{11}{|c|}{ Panel B. $[\alpha+(1-\alpha) \beta]=0.5$} \\
\hline$\beta$ & $\gamma$ & $\alpha$ & & & & & & & & \\
\hline 0.00 & 0.020 & 0.50 & $\begin{array}{c}6.23 \\
(3.00)\end{array}$ & $\begin{array}{c}5.12 \\
(3.00)\end{array}$ & $\begin{array}{c}4.23 \\
(3.00)\end{array}$ & $\begin{array}{c}3.81 \\
(3.00)\end{array}$ & 0.055 & 0.38 & 0.21 & 0.0 \\
\hline 0.14 & 0.016 & 0.42 & 2.53 & 2.42 & 2.29 & 2.21 & 0.055 & 0.32 & 0.25 & 0.0 \\
\hline 0.24 & 0.013 & 0.34 & 2.24 & 2.12 & 1.97 & 1.89 & 0.055 & 0.26 & 0.31 & 0.0 \\
\hline 0.32 & 0.010 & 0.26 & 2.03 & 1.90 & 1.77 & 1.69 & 0.055 & 0.20 & 0.40 & 0.0 \\
\hline 0.39 & 0.008 & 0.18 & 1.86 & 1.74 & 1.61 & 1.54 & 0.055 & 0.14 & 0.58 & 0.0 \\
\hline 0.44 & 0.007 & 0.10 & 1.73 & 1.61 & 1.49 & 1.43 & 0.055 & 0.08 & 1.05 & 0.0 \\
\hline
\end{tabular}

Note: $\lambda=0, \psi=0$. 
TABLE D

Speed of Convergence as the Disembodied learning Parameter, $\beta$, RISES AND $\psi$ IS ADJUSTED SO AS TO MAINTAIN $g_{c}^{*}=0.02$

\begin{tabular}{|c|c|c|c|c|c|c|c|c|c|c|}
\hline & & & \multicolumn{4}{|c|}{ Speed of Convergence in \% } & \multicolumn{4}{|c|}{$\theta=1.75$} \\
\hline & & & $\theta=1$ & $\theta=1.75$ & $\theta=3$ & $\theta=4$ & $r^{*}$ & $s^{*}$ & $(Y /(p K))^{*}$ & $g_{p}^{*}$ \\
\hline \multicolumn{11}{|c|}{ PANEL A. } \\
\hline$\beta$ & $\psi$ & $\alpha$ & & & & & & & & \\
\hline 0.00 & 0.042 & 0.32 & $\begin{array}{c}15.4 \\
(3.00)\end{array}$ & $\begin{array}{c}12.5 \\
(3.00)\end{array}$ & $\begin{array}{c}10.3 \\
(3.00)\end{array}$ & $\begin{array}{c}9.33 \\
(3.00)\end{array}$ & 0.055 & 0.27 & 0.45 & -0.042 \\
\hline 0.13 & 0.033 & 0.32 & 2.58 & 2.52 & 2.43 & 2.38 & 0.055 & 0.27 & 0.43 & -0.033 \\
\hline 0.27 & 0.025 & 0.32 & 2.16 & 2.05 & 1.92 & 1.85 & 0.055 & 0.26 & 0.40 & -0.025 \\
\hline 0.40 & 0.017 & 0.32 & 1.75 & 1.62 & 1.47 & 1.39 & 0.055 & 0.26 & 0.38 & -0.017 \\
\hline 0.53 & 0.008 & 0.32 & 1.34 & 1.20 & 1.06 & 1.00 & 0.055 & 0.25 & 0.35 & -0.008 \\
\hline 0.67 & 0.000 & 0.32 & 0.95 & 0.82 & 0.71 & 0.66 & 0.055 & 0.25 & 0.32 & 0.000 \\
\hline \multicolumn{11}{|c|}{ Panel B. $[\alpha+(1-\alpha) \beta]=0.5$} \\
\hline$\beta$ & $\psi$ & $\alpha$ & & & & & & & & \\
\hline 0.00 & 0.020 & 0.50 & $\begin{array}{c}7.64 \\
(3.00)\end{array}$ & $\begin{array}{c}6.18 \\
(3.00)\end{array}$ & $\begin{array}{c}5.00 \\
(3.00)\end{array}$ & $\begin{array}{c}4.45 \\
(3.00)\end{array}$ & 0.055 & 0.40 & 0.25 & -0.020 \\
\hline 0.14 & 0.022 & 0.42 & 2.54 & 2.45 & 2.33 & 2.25 & 0.055 & 0.34 & 0.30 & -0.022 \\
\hline 0.24 & 0.025 & 0.34 & 2.23 & 2.13 & 2.00 & 1.92 & 0.055 & 0.28 & 0.38 & -0.025 \\
\hline 0.32 & 0.029 & 0.26 & 2.01 & 1.90 & 1.78 & 1.71 & 0.055 & 0.21 & 0.52 & -0.029 \\
\hline 0.39 & 0.038 & 0.18 & 1.83 & 1.73 & 1.62 & 1.55 & 0.055 & 0.15 & 0.79 & -0.038 \\
\hline 0.44 & 0.060 & 0.10 & 1.68 & 1.59 & 1.49 & 1.43 & 0.055 & 0.09 & 0.65 & -0.060 \\
\hline
\end{tabular}

Note: $\lambda=0, \gamma=0$.

TABLE E

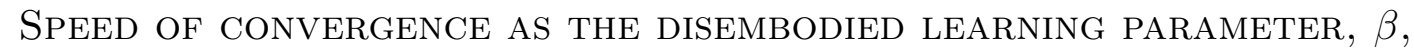
RISES AND $\lambda$ IS ADJUSTED SO AS TO MAINTAIN $g_{c}^{*}=0.02$

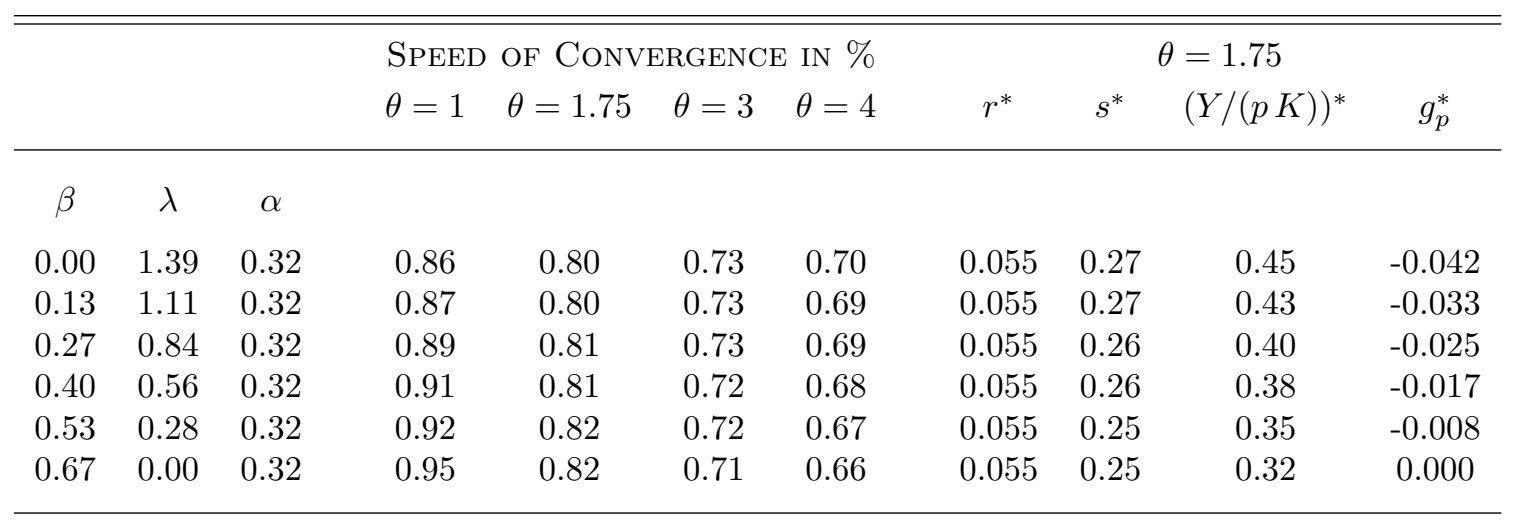

Note: $\gamma=0, \psi=0$. 
TABLE F

SPEED OF CONVERGENCE AS THE EXOGENOUS EMBODIED CHANGE

PARAMETER, $\psi$, RISES AND $\gamma$ IS ADJUSTED SO AS TO MAINTAIN $g_{c}^{*}=0.02$

\begin{tabular}{|c|c|c|c|c|c|c|c|c|c|c|}
\hline & & \multicolumn{5}{|c|}{ Speed of Convergence of $(x, z)$ In $\%$} & \multicolumn{4}{|c|}{$\theta=1.75$} \\
\hline & & & $\theta=1$ & $\theta=1.75$ & $\theta=3$ & $\theta=4$ & $r^{*}$ & $s^{*}$ & $(Y /(p K))^{*}$ & $g_{p}^{*}$ \\
\hline$\psi$ & $\gamma$ & $\alpha$ & & & & & & & & \\
\hline 0.000 & 0.020 & 0.32 & 10.48 & 8.78 & 7.52 & 6.96 & 0.055 & 0.25 & 0.32 & 0.000 \\
\hline 0.008 & 0.016 & 0.32 & 11.47 & 9.52 & 8.08 & 7.43 & 0.055 & 0.25 & 0.35 & -0.008 \\
\hline 0.017 & 0.012 & 0.32 & 12.46 & 10.27 & 8.63 & 7.90 & 0.055 & 0.26 & 0.38 & -0.017 \\
\hline 0.025 & 0.008 & 0.32 & 13.45 & 11.01 & 9.19 & 8.37 & 0.055 & 0.26 & 0.40 & -0.025 \\
\hline 0.033 & 0.004 & 0.32 & 14.44 & 11.76 & 9.75 & 8.85 & 0.055 & 0.27 & 0.43 & -0.033 \\
\hline 0.042 & 0.000 & 0.32 & 15.43 & 12.51 & 10.32 & 9.33 & 0.055 & 0.27 & 0.45 & -0.042 \\
\hline
\end{tabular}

Note: $\beta=0, \lambda=0$.

\section{References}

[1] Acemoglu, D. (2009), Introduction to Modern Economic Growth, Princeton: Princeton University Press.

[2] Aghion, P., P. Howitt (1998), Endogenous Growth Theory, Cambridge (Mass.): MIT Press.

[3] Aghion, P., P. Howitt (2009), The Economics of Growth, Cambridge (Mass.): MIT Press.

[4] Arrow, K. J. (1962), The Economic Implications of Learning by Doing. Review of Economic Studies 29, 153-73.

[5] Barro, R. J., X. Sala-i-Martin (1992), Convergence, Journal of Political Economy 100, 223-251.

[6] Barro, R. J., X. Sala-i-Martin (2004), Economic Growth, 2nd ed., Cambridge (Mass.): MIT Press.

[7] Boucekkine, R., F. del Rio, O. Licandro (2003), Embodied Technological Change, Learning-by-doing and the Productivity Slowdown. Scandinavian Journal of Economics 105 (1), 87-97. 
[8] Boucekkine, R., T. Ruiz-Tamarit (2004), Imbalance Effects in the Lucas model: An Analytical Exploration, Topics in Macroeconomics 4 (1) Article 15.

[9] Chatterjee, S. (2005), Capital Utilization, Economic Growth and Convergence, Journal of Economic Dynamics and Development 29, 2093-2124.

[10] de la Croix, D. P. Michel, A Theory of Economic Growth, Cambridge, UK: Cambridge University Press.

[11] Eicher, T., S. J. Turnovsky (1999), Convergence Speeds and Transitional Dynamics in Non-Scale Growth Models, Journal of Economic Growth 4, 413-428.

[12] Evans, P. (1997), How Fast do Economies Converge?, Review of Economics and Statistics 79, 219-225.

[13] Greenwood, J., Z. Hercowitz, P. Krusell (1997), Long-Run Implications of Investment-Specific Technological Change. American Economic Review 87 (3), $342-362$.

[14] Greenwood, J., B. Jovanovic (2001), Accounting for growth. In: New Developments in Productivity Analysis, ed. by C. R. Hulten, E. R. Dean, M. J. Harper, NBER Studies in Income and Wealth, Chicago: University of Chicago Press.

[15] Groth, C. (2010), Embodied Learning and Growth: The Simple Analytics, Working Paper, Department of Economics, University of Copenhagen.

[16] Groth, C., K.-J. Koch, T. M. Steger (2010), When Economic Growth is Less Than Exponential, Economic Theory 44, 213-242.

[17] Hornstein, A., P. Krusell (1996), Can technology improvements cause productivity slowdowns? NBER Macroeconomics Annual 11, 209-259.

[18] Hornstein, A., P. Krusell, G. L. Violante (2005), The Effect of Technical Change on Labor Market Inequalities. In: Handbook of Economic Growth, vol. 1B, ed. by P. Aghion, and S. N. Durlauf, Amsterdam: Elsevier, 1275-1370. 
[19] Islam, N. (1995), Growth Empirics. A Panel Data Approach, Quarterly Journal of Economics 110, 1127-1170.

[20] Jovanovic, B. (1997), Learning and Growth. In: Advances in Economics and Econometrics: Theory and Applications, vol. II, ed. by D. M. Kreps and K. F. Wallis, Cambridge: Cambridge University Press, 318-339.

[21] Jovanovic, B., P. L. Rousseau (2002), Moore's Law and Learning by Doing, Review of Economic Dynamics 5, 346-375.

[22] Levine, R., D. Renelt (1992), A Sensitivity Analysis of Cross-Country Growth Regressions, American Economic Review 82, 942-963.

[23] Mankiw, N. G., D. Romer, D. Weil (1992), A Contribution to the Empirics of Economic Growth, Quarterly Journal of Economics 107, 407-438.

[24] McQinn, K., K. Whelan (2007), Conditional Convergence and the Dynamics of the Capital-Output Ratio, Journal of Economic Growth 12, 159-184.

[25] Ortigueira, S., M. S. Santos (1997), On the Speed of Convergence in Endogenous Growth Models, American Economic Review 87, 383 - 399.

[26] Phelps, E. (1962), The New View of Investment: A Neoclassical Analysis, Quarterly Journal of Economics 76 (4), 548-567.

[27] Romer, P. M. (1986), Increasing Returns and Long-run Growth, Journal of Political Economy 94, 1002-1037.

[28] Sakellaris, P., D. J. Wilson (2004), Quantifying Embodied Technological Change, Review of Economic Dynamics 7, 1-26.

[29] Solow, R. M. (1960), Investment and Technical Progress. In: K. J. Arrow, S. Karlin, and P. Suppes, eds., Mathematical Methods in the Social Sciences, Stanford: Stanford University Press, pp. 89-104. 
[30] Turnovsky, S. J. (2002), Intertemporal and Intratemporal Substitution, and the Speed of Convergence in the Neoclassical Growth Model, Journal of Economic Dynamics and Control 26, 1765-1785.

[31] Xie, D. (1994), Divergence in Economic Performance: Transitional Dynamics with Multiple Equilibria, Journal of Economic Theory 63, 97-112.

[32] Valdés, B. (1999), Economic Growth. Theory, Empirics and Policy, Cheltenham, UK: Edward Elgar.

[33] Williams, R. L., R. L. Crouch (1972), The Adjustment Speed of Neoclassical Growth Models, Journal of Economic Theory 4, 552-556. 